This is a postprint version of the following published document:

Elvira, Víctor; Míguez, Joaquín; Djuri'c, Petar M. Adapting the number of particles in sequential Monte Carlo methods through an online scheme for convergence assessment. IEEE Transactions on Signal Processing (2017), 65(7), $1781-1794$.

DOI: https://doi.org/10.1109/TSP.2016.2637324

(C) 2016 IEEE. Personal use of this material is permitted. Permission from IEEE must be obtained for all other uses, in any current or future media, including reprinting/republishing this material for advertising or promotional purposes, creating new collective works, for resale or redistribution to servers or lists, or reuse of any copyrighted component of this work in other works. 


\title{
Adapting the Number of Particles in Sequential Monte Carlo Methods Through an Online Scheme for Convergence Assessment
}

\author{
Víctor Elvira, Member, IEEE, Joaquín Míguez, and Petar M. Djurić, Fellow, IEEE
}

\begin{abstract}
Particle filter are broadly used to approximate posterior distributions of hidden states in state-space models by means of sets of weighted particles. While the convergence of the filte is guaranteed when the number of particles tends to infinit, the quality of the approximation is usually unknown but strongly dependent on the number of particles. In this paper, we propose a novel method for assessing the convergence of particle filter in an online manner, as well as a simple scheme for the online adaptation of the number of particles based on the convergence assessment. The method is based on a sequential comparison between the actual observations and their predictive probability distributions approximated by the filte. We provide a rigorous theoretical analysis of the proposed methodology and, as an example of its practical use, we present simulations of a simple algorithm for the dynamic and online adaptation of the number of particles during the operation of a particle filte on a stochastic version of the Lorenz 63 system.
\end{abstract}

Index Terms-Particle filtering sequential Monte Carlo, convergence assessment, predictive distribution, convergence analysis, computational complexity, adaptive complexity.

\section{INTRODUCTION}

\section{A. Background}

M ANY problems in science and engineering can be described by dynamical models where hidden states of the systems change over time and observations that are functions of the states are available. Often, the observations are sequentially

This work was supported in part by the Ministerio de Economía y Competitividad of Spain under Grant TEC2013-41718-R OTOSiS, Grant TEC2012-38883-C02-01 COMPREHENSION, and Grant TEC2015-69868C2-1-R ADVENTURE, in part by the Offic of Naval Research Global under Grant N62909-15-1-2011, and in part by the National Science Foundation under Grant CCF-1320626 and Grant CCF-1618999. acquired and the interest is in making recursive inference on the hidden states. In many applications, the Bayesian approach to the problem is adopted because it allows for optimal inclusion of prior knowledge of the unknown state in the estimation process [1], [2]. In this case, the prior information and the likelihood function that relates the hidden state and the observation are combined yielding a posterior distribution of the state.

Exact Bayesian inference, however, is only possible in a small number of scenarios, including linear Gaussian state-space models (using the Kalman filte [3], [4]) and finit state-space hidden Markov models (HMM filter [5]). Therefore, in many other practical problems, only approximate inference methods can be used. One class of suboptimal methods is particle filtering which is also known as sequential Monte Carlo sampling [6] [10]. Since the publication of [11], where the sampling importance resampling (SIR) filte was introduced, particle filterin has received outstanding attention in research and practice. Particle filter approximate posterior distributions of the hidden states sequentially and recursively. They do it by exploiting the principle of importance sampling and by using sets of weighted particles [6], [7], [12].

One key parameter of particle filter is the number of particles. It can be proved that the rate of convergence of the approximate probability distribution towards the true posterior is inversely proportional to the square root of the number of particles used in the filte [12], [13]. This, too, entails that the filte "perfectly" approximates the posterior distribution when the number of particles tends to infinit. However, since the computational cost grows with the number of particles, practitioners must choose a specifi number of particles in the design of their filters

In many applications, the observations arrive sequentially, and there is a strict deadline for processing each new observation. Then, one could argue that the best solution in terms of filte performance is to increase the number of particles as much as possible and keep it fi ed. Also, in some hardware implementations, the number of particles is a design parameter that cannot be modifie during implementation. Nevertheless, in many other applications where resources are scarce or are shared with a dynamical allocation and/or with energy restrictions, one might be interested in adapting the number of particles in a smart way. One would use enough particles to achieve a certain performance requirement but without wasting resources by using many more particles if they do not translate into a significan improvement of the filte performance. 
The selection of the number of particles, however, is often a delicate subject because, (1) the performance of the fil ter (the quality of the approximation) cannot usually be described in advance as a function of the number of particles, and (2) the mismatch between the approximation provided by the filte and the unknown posterior distribution is obviously also unknown. Therefore, although there is a clear trade-off between performance and computational cost, this relation is not straightforward; e.g., increasing the number of particles over a certain value may not significantl improve the quality of the approximation while decreasing the number of particles below some other value can dramatically affect the performance of the filte.

Few papers in the wide literature have addressed the problem of online assessment of the filte convergence for the purpose of adapting the number of particles. In [14], the number of particles is selected so that a bound on the approximation error does not exceed a threshold with certain probability. The latter error is define as the Kullback-Leibler divergence (KLD) between the approximate filte distribution and a grid-discretized version of the true one (which is itself a potentially-costly approximation with an unknown error). In [15], an adaptation of the number of particles is proposed, based on the KLD approach of [14] and an estimate of the variance of the estimators computed via the particle filte, along with an improvement of the proposal distributions. In [16], the adaptation of the number of particles is based on the effective sample size. These methods are heuristic: they do not enjoy any theoretical guarantees (in the assessment of the approximation errors made by the particle filter and the allocation of particles, therefore, cannot be ensured to be optimal according to any probabilistic criterion. Some techniques based on more solid theoretical ground have been proposed, within the applied probability community, during the last few years. We discuss them below.

Two types of unbiased estimators of the variance in the approximation of integrals using a class of particle filter were analyzed in [17] using the Feynman-Kac framework of [18]. As an application of these results, it was suggested to use these estimators to select the number of particles in the filte . In particular, the scheme proposed in [17] is a batch procedure in which a particle filte is run several times over the whole data sequence, with increasing number of particles, until the variance of the integral of interest is found to fall below a prescribed threshold. This approach cannot be used for online assessment, which is the goal of the present paper. Another batch method (thus, also not applicable for online assessment) for particle allocation has been recently proposed in [19], where an ad hoc autoregressive model is fitte to estimate the variance of the estimators produced by the particle filte .

Papers on so-called alive particle filter can also be found in the literature [20]-[22]. These articles focus on models where the likelihood function can take zero value for some regions of the state space, in such a way that there is the risk that a collection of zero-weight particles are generated if a standard algorithm is employed. To avoid this limitation, alive particle filter are based on sampling schemes where new particles are generated until a prescribed number of them, $M$, attain non-zero weights. The computational cost of the algorithm per time step is, therefore, random. Moreover, the number $M$ is chosen a priori and there is no assessment of whether $M$ allows for reaching adequate accuracy of the estimators (the methodology proposed in the present manuscript can be directly applied to alive particle filter in order to adapt $M$ ).

In order to guarantee that the particle set yields a sufficientl good representation, in [23] it is proposed to test whether the particle estimate of the predictive density of the observation at time $t$ given the previous data is sufficientl large, i.e., whether it is above a prescribed (heuristically chosen) threshold. When the particle set does not satisfy this condition, it is discarded and a new collection of particles is generated. The number of particles is not adapted, since all generated sets have the same size. The computational cost of this algorithm is random.

Finally, in [24, Chapter 4] it is proposed to use the coeffi cient of variation of the weights (or, equivalently, the effective sample size) in order to detect those observations for which there is a large $\chi^{2}$-divergence between the proposal distribution used to generate the set of particles and the target distribution. This connection is rigorously established in [24]. The algorithm, however, is computationally costly compared to classical methods: at each time step, a complete set of particles and weights are generated, and the coefficien of variation is computed. If this coefficien is too high, the particles are discarded, the algorithm "rolls back," and a new, larger set of particles is generated for better representation of the target distribution (this step is termed "refuelling" in [24]). Although the algorithm enjoys theoretical guarantees, it relies on keeping the particle approximation "locked" to the target distribution at all times. It is known that, once the particle filte has lost track of the state distribution, the effective sample size (and, hence, coefficien of variation) becomes uninformative [25] and, therefore, the link with the $\chi^{2}$-divergence is lost.

\section{B. Contributions}

We introduce a model-independent methodology for the online assessment of the convergence of particle filter and carry out a rigorous analysis that ensures the consistency of the proposed scheme under fairly standard assumptions. The method is an extension of our previous work presented in [26]. In the proposed scheme, the observations are processed one at a time and the filte performance is assessed by measuring the discrepancy between the actual observation at each time step and a number of fictitiou data-points drawn from the particle approximation of the predictive probability distribution of the observations. The method can be exploited to adjust the number of particles dynamically when the performance of the filte degrades below a certain desired level. This would allow a practitioner to select the operation point by considering performance-computational cost tradeoffs. Based on the method, we propose a simple and efficien algorithm that adjusts the number of particles in real time. We demonstrate the performance of the algorithm numerically by running it for a stochastic version of the 3-dimensional Lorenz 63 system. As already noted, this paper builds on the method from [26]. However, the main difference here is that the underlying model is not questioned - instead, it is assumed 
to be correct. The connection between [26] and the present work is that they both build upon the ability to compute predictive statistics of the upcoming observations that turn out to be independent of the underlying state space model. In this paper we have rigorous theoretical results regarding the particle approximations of the predictive distribution of the observations (while this issue was ignored in [26]). Finally, we suggest practical schemes for the online adjustment of the number of particles.

Let us point out that the adaptive procedure for the online selection of the number of particles described herein is only one of many that can exploit the results of the convergence analysis. In other words, our analysis opens the door for development of new families of algorithms for online adaptation of the number of particles by way of online convergence assessment.

\section{Organization of the Paper}

The rest of the paper is organized as follows. In Section II we describe the class of state space Markov models and provide a basic background on the well-known bootstrap particle filte of [11]. The theoretical results that enable the online assessment of particle filter are stated in Section III, with full details and proofs contained in the Supplementary Material. The proposed methodology for online convergence assessment of the particle filte is introduced in Section IV. Furthermore, this section provides a simple algorithm for the dynamic, online adaptation of the number of particles. In Section V, we illustrate the validity of the method by means of computer simulations for a stochastic Lorenz 63 model. Finally, Section VI contains a summary of results and some concluding remarks.

\section{PARTICLE FiLTERING}

In this section we describe the class of state space models of interest and then present the standard particle filte (PF), which is the basic building block for the methods to be introduced later.

\section{A. State Space Models and Stochastic Filtering}

Let us consider discrete-time, Markov dynamic systems in state-space form described by the triplet ${ }^{1}$

$$
\begin{aligned}
\boldsymbol{X}_{0} & \sim p\left(\boldsymbol{x}_{0}\right), \\
\boldsymbol{X}_{t} & \sim p\left(\boldsymbol{x}_{t} \mid \boldsymbol{x}_{t-1}\right), \\
\boldsymbol{Y}_{t} & \sim p\left(\boldsymbol{y}_{t} \mid \boldsymbol{x}_{t}\right),
\end{aligned}
$$

where

- $t \in \mathbb{N}$ denotes discrete time;

- $\boldsymbol{X}_{t}$ is the $d_{x} \times 1$-dimensional (random) system state at time $t$, which takes variables in the set $\mathcal{X} \subseteq \mathbb{R}^{d_{x}}$,

\footnotetext{
${ }^{1}$ In most of the paper we abide by a simplifie notation where $p(x)$ denotes the probability density function (pdf) of the random variable $X$. This notation is argument-wise, hence if we have two random variables $X$ and $Y$, then $p(x)$ and $p(y)$ denote the corresponding density functions, possibly different; $p(x, y)$ denotes the joint pdf and $p(x \mid y)$ is the conditional pdf of $X$ given $Y=y$. A more accurate notation, which avoids ambiguities, is used for the analysis and the statement of the theoretical results. Besides, vectors are denoted by bold-face letters, e.g., $\boldsymbol{x}$, while regular-face is used for scalars, e.g., $x$.
}

- $p\left(\boldsymbol{x}_{0}\right)$ is the a priori pdf of the state, while

- $p\left(\boldsymbol{x}_{t} \mid \boldsymbol{x}_{t-1}\right)$ denotes the conditional density of the state $\boldsymbol{X}_{t}$ given $\boldsymbol{X}_{t-1}=\boldsymbol{x}_{t-1}$

- $\boldsymbol{Y}_{t}$ is the $d_{y} \times 1$-dimensional observation vector at time $t$, which takes values in the set $\mathcal{Y} \subseteq \mathbb{R}^{d_{y}}$ and is assumed to be conditionally independent of all other observations given the state $\boldsymbol{X}_{t}$,

- $p\left(\boldsymbol{y}_{t} \mid \boldsymbol{x}_{t}\right)$ is the conditional pdf of $\boldsymbol{Y}_{t}$ given $\boldsymbol{X}_{t}=\boldsymbol{x}_{t}$. It is often referred to as the likelihood of $\boldsymbol{x}_{t}$, when it is viewed as a function of $\boldsymbol{x}_{t}$ given $\boldsymbol{y}_{t}$.

The model described by Eqs. (1)-(3) includes a broad class of systems, both linear and nonlinear, with Gaussian or nonGaussian perturbations. Here we focus on the case where all the model parameters are known. However, the proposed method can also be used for models with unknown parameters for which suitable particle filterin methods are available [27]-[29]. We assume that the prior distribution of the state $p\left(\boldsymbol{x}_{0}\right)$ is also known.

The stochastic filterin problem consists in the computation of the sequence of posterior probability distributions given by the so-called filterin densities $p\left(\boldsymbol{x}_{t} \mid \boldsymbol{y}_{1: t}\right), t=1,2, \cdots$. The pdf $p\left(\boldsymbol{x}_{t} \mid \boldsymbol{y}_{1 \cdot t}\right)$ is closely related to the one-step-ahead predictive state density $p\left(\boldsymbol{x}_{t} \mid \boldsymbol{y}_{1: t-1}\right)$, which is of major interest in many applications and can be written down by way of the ChapmanKolmogorov equation,

$$
p\left(\boldsymbol{x}_{t} \mid \boldsymbol{y}_{1: t-1}\right)=\int p\left(\boldsymbol{x}_{t} \mid \boldsymbol{x}_{t-1}\right) p\left(\boldsymbol{x}_{t-1} \mid \boldsymbol{y}_{1: t-1}\right) d \boldsymbol{x}_{t-1} .
$$

Using Bayes' theorem together with Eq. (4), we obtain the wellknown recursive factorization of the filterin pdf

$$
p\left(\boldsymbol{x}_{t} \mid \boldsymbol{y}_{1: t}\right) \propto p\left(\boldsymbol{y}_{t} \mid \boldsymbol{x}_{t}\right) \int p\left(\boldsymbol{x}_{t} \mid \boldsymbol{x}_{t-1}\right) p\left(\boldsymbol{x}_{t-1} \mid \boldsymbol{y}_{1: t-1}\right) d \boldsymbol{x}_{t-1} .
$$

For conciseness and notational accuracy, we use the measuretheoretic notation

$$
\pi_{t}\left(d \boldsymbol{x}_{t}\right):=p\left(\boldsymbol{x}_{t} \mid \boldsymbol{y}_{1: t}\right) d \boldsymbol{x}_{t}, \quad \xi_{t}\left(d \boldsymbol{x}_{t}\right):=p\left(\boldsymbol{x}_{t} \mid \boldsymbol{y}_{1: t-1}\right) d \boldsymbol{x}_{t}
$$

to represent the filterin and the predictive posterior probability distributions of the state, respectively. Note that $\pi_{t}$ and $\xi_{t}$ are probability measures, hence, given a Borel set $A \subset \mathcal{X}$, $\pi_{t}(A)=\int_{A} \pi\left(d \boldsymbol{x}_{t}\right)$ and $\xi_{t}(A)=\int_{A} \xi_{t}\left(d \boldsymbol{x}_{t}\right)$ denote the posterior probability of the event $\boldsymbol{X}_{t} \in A$ conditional on $\boldsymbol{Y}_{1: t}=\boldsymbol{y}_{1: t}$ and $\boldsymbol{Y}_{1: t-1}=\boldsymbol{y}_{1: t-1}$, respectively.

However, the object of main interest for the convergence assessment method to be introduced in this paper is the predictive pdf of the observations, namely the function $p\left(\boldsymbol{y}_{t} \mid \boldsymbol{y}_{1: t-1}\right)$ and the associated probability measure

$$
\mu_{t}\left(d \boldsymbol{y}_{t}\right):=p\left(\boldsymbol{y}_{t} \mid \boldsymbol{y}_{1: t-1}\right) d \boldsymbol{y}_{t} .
$$

The density $p\left(\boldsymbol{y}_{t} \mid \boldsymbol{y}_{1: t-1}\right)$ is the normalization constant of the filterin density $p\left(\boldsymbol{x}_{t} \mid \boldsymbol{y}_{1: t}\right)$, and it is related to the predictive state $\operatorname{pdf} p\left(\boldsymbol{x}_{t} \mid \boldsymbol{y}_{1: t-1}\right)$ through the integral

$$
p\left(\boldsymbol{y}_{t} \mid \boldsymbol{y}_{1: t-1}\right)=\int p\left(\boldsymbol{y}_{t} \mid \boldsymbol{x}_{t}\right) p\left(\boldsymbol{x}_{t} \mid \boldsymbol{y}_{1: t-1}\right) d \boldsymbol{x}_{t} .
$$

It also plays a key role in model assessment [26] and model inference problems [28]-[30], [31]. 


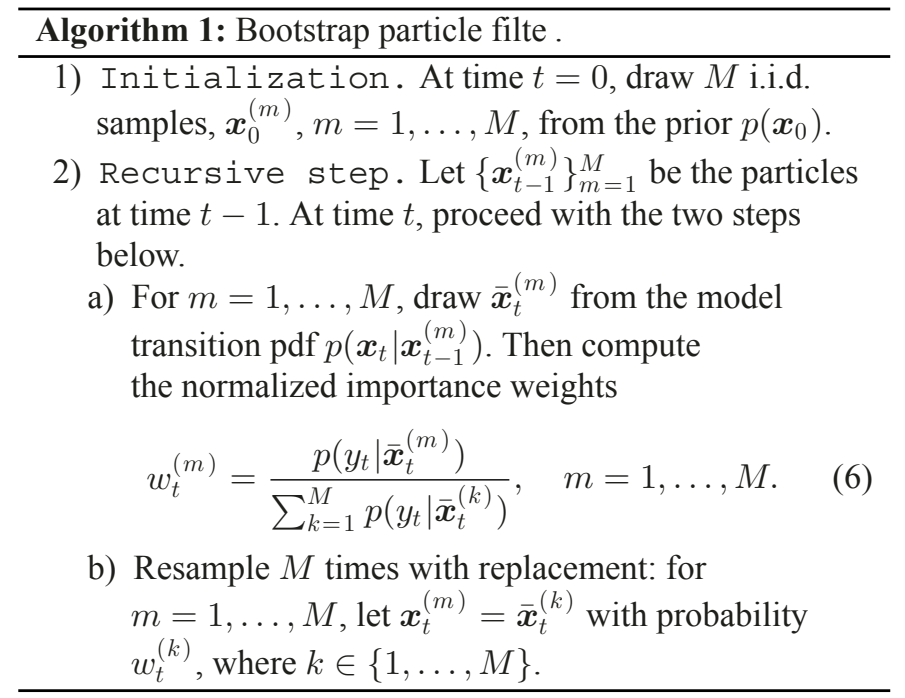

\section{B. The Standard Particle Filter}

A PF is an algorithm that processes the observations $\left\{\boldsymbol{y}_{t}\right\}_{t \geq 1}$ sequentially in order to compute Monte Carlo approximations of the sequence of probability measures $\left\{\pi_{t}\right\}_{t \geq 1}$. The simplest algorithm is the so-called bootstrap particle filte (BPF) [11] (see also [32]), which consists of a recursive importance sampling procedure and a resampling step. The term "particle" refers to a Monte Carlo sample in the state space $\mathcal{X}$, which is assigned an importance weight. Below, we outline the BPF algorithm with $M$ particles.

For the sake of simplicity, in step 2. (b) above we assume that multinomial resampling [7] is carried out for every $t \geq 1$. The results and methods to be presented in subsequent sections remain valid when resampling is carried out periodically and/or using alternative schemes such as residual [6], stratifie [33] or minimum-variance [34] resampling (see also [35]).

The simple BPF yields several useful approximations. After sampling at step 2. (a), the predictive state probability measure $\xi_{t}$ can be approximated as

$$
\xi_{t}^{M}\left(d \boldsymbol{x}_{t}\right)=\frac{1}{M} \sum_{m=1}^{M} \delta_{\overline{\boldsymbol{x}}_{t}^{(m)}}\left(d \boldsymbol{x}_{t}\right),
$$

where $\delta_{x}$ denotes the Dirac delta measure located at $x \in \mathcal{X}$. The filte measure $\pi_{t}$ can be similarly approximated, either using the particles and weights computed at step 2. (a) or the resampled particles after step 2. (b), i.e.,

$$
\bar{\pi}_{t}^{M}=\sum_{m=1}^{M} w_{t}^{(m)} \delta_{\overline{\boldsymbol{x}}_{t}^{(m)}} \quad \text { and } \quad \pi_{t}^{M}=\frac{1}{M} \sum_{m=1}^{M} \delta_{\boldsymbol{x}_{t}^{(m)}},
$$

respectively. In addition, the BPF yields natural approximations of the predictive pdf's of $\boldsymbol{X}_{t}$ and $\boldsymbol{Y}_{t}$ given the earlier observations $\boldsymbol{Y}_{1: t-1}=\boldsymbol{y}_{1: t-1}$. If we specificall denote these functions as $\tilde{p}_{t}\left(\boldsymbol{x}_{t}\right):=p\left(\boldsymbol{x}_{t} \mid \boldsymbol{y}_{1: t-1}\right)$ and $p_{t}\left(\boldsymbol{y}_{t}\right):=p\left(\boldsymbol{y}_{t} \mid \boldsymbol{y}_{1: t-1}\right)$, then we readily obtain their respective estimates as mixture distributions with $M$ mixands, or,

$$
\begin{aligned}
& \tilde{p}_{t}^{M}\left(\boldsymbol{x}_{t}\right):=\sum_{m=1}^{M} w_{t-1}^{M} p\left(\boldsymbol{x}_{t} \mid \boldsymbol{x}_{t-1}^{(m)}\right), \quad \text { and } \\
& p_{t}^{M}\left(\boldsymbol{y}_{t}\right):=\frac{1}{M} \sum_{m=1}^{M} p\left(\boldsymbol{y}_{t} \mid \overline{\boldsymbol{x}}_{t}^{(m)}\right),
\end{aligned}
$$

for any $\boldsymbol{x}_{t} \in \mathcal{X}$ and $\boldsymbol{y}_{t} \in \mathcal{Y}$.

\section{A Novel Asymptotic Convergence Result}

The convergence of the approximate measures, e.g., $\xi_{t}^{M}$, towards the true ones is usually assessed in terms of the estimates of 1-dimensional statistics of the corresponding probability distribution. To be specific let $f: \mathcal{X} \rightarrow \mathbb{R}$ be a real integrable function in the state space and denote ${ }^{2}$

$$
\left(f, \xi_{t}\right):=\int f\left(\boldsymbol{x}_{t}\right) \xi_{t}\left(d \boldsymbol{x}_{t}\right)
$$

Under mild assumptions on the state space model, it can be proved that

$$
\lim _{M \rightarrow \infty}\left(f, \xi_{t}^{M}\right)=\lim _{M \rightarrow \infty} \frac{1}{M} \sum_{m=1}^{M} f\left(\boldsymbol{x}_{t}^{(m)}\right)=\left(f, \xi_{t}\right)
$$

almost surely (a.s.) [12], [18].

According to (5), the predictive observation pdf $p_{t}\left(\boldsymbol{y}_{t}\right)$ is an integral w.r.t. $\xi_{t}$ and, as a consequence, Eq. (7) implies that $\lim _{M \rightarrow \infty} p_{t}^{M}(\boldsymbol{y})=p_{t}(\boldsymbol{y})$ a.s. and point-wise for every $\boldsymbol{y} \in \mathcal{Y}$ under mild assumptions [18]. However, existing theoretical results do not ensure that $p_{t}^{M}(\boldsymbol{y})$ can converge uniformly on $\mathcal{Y}$ towards $p_{t}(\boldsymbol{y})$ and this fact prevents us from claiming that $\lim _{M \rightarrow \infty} \int h(\boldsymbol{y}) p_{t}^{M}(\boldsymbol{y}) d \boldsymbol{y}=\int h(\boldsymbol{y}) p_{t}(\boldsymbol{y}) d \boldsymbol{y}=\left(h, \mu_{t}\right)$ in some proper sense for integrable real functions $h(\boldsymbol{y})$.

Important contributions of this paper are (a) the proof of a.s. convergence of the random probability measure

$$
\mu_{t}^{M}(d \boldsymbol{y}):=p_{t}^{M}(\boldsymbol{y}) d \boldsymbol{y}
$$

towards $\mu_{t}$ (as $M \rightarrow \infty$ ) under mild regularity assumptions on the state space model, and (b) the provision of explicit error rates. We point out that $\mu_{t}^{M}$ is not a classical point-mass Monte Carlo approximation of $\mu_{t}$ (as, for example, $\pi_{t}^{M}$ is an approximation of $\pi_{t}$ ). Instead, the measure $\mu_{t}^{M}$ is absolutely continuous with respect to the Lebesgue measure (the same as $\mu_{t}$ itself). If a different reference measure were used to defin the pdf's $p\left(\boldsymbol{x}_{t} \mid \boldsymbol{x}_{t-1}\right)$ and $p\left(\boldsymbol{y}_{t} \mid \boldsymbol{x}_{t}\right)$, say $\nu$, then both $\mu_{t}$ and $\mu_{t}^{M}$ would be absolutely continuous with respect to $\nu$. In order to describe how $\mu_{t}^{M}$ converges to $\mu_{t}$ in a rigorous manner, we need to introduce some notation:

- For each $t \geq 1$, let us defin the function $g_{t}\left(\boldsymbol{y}_{t}, \boldsymbol{x}_{t}\right):=$ $p\left(\boldsymbol{y}_{t} \mid \boldsymbol{x}_{t}\right)$, i.e., the conditional pdf of $\boldsymbol{y}_{t}$ given $\boldsymbol{x}_{t}$. When this function is used as a likelihood, we write $g_{t}^{\boldsymbol{y}_{t}}\left(\boldsymbol{x}_{t}\right):=$ $g_{t}\left(\boldsymbol{y}_{t}, \boldsymbol{x}_{t}\right)$ to emphasize that it is a function of $\boldsymbol{x}_{t}$.

${ }^{2}$ Let $(\mathcal{Z}, \mathcal{B}(\mathcal{Z}))$ be a measurable space, where $\mathcal{Z} \subseteq \mathbb{R}^{d}$ for some integer $d \geq 1$ and $\mathcal{B}(\mathcal{Z})$ is the Borel $\sigma$-algebra of subsets of $\mathcal{Z}$. If $\alpha$ is a measure on $\mathcal{B}(\mathcal{Z})$ and the function $h: \mathcal{Z} \rightarrow \mathbb{R}$ is integrable with respect to (w.r.t.) $\alpha$, then we use the shorthand notation $(f, \alpha):=\int f(z) \alpha(d z)$. 
- Let $f: \mathcal{Z} \rightarrow \mathbb{R}$ be a real function on some set $\mathcal{Z}$. We denote the absolute supremum of $f$ as $\|f\|_{\infty}:=\sup _{\boldsymbol{z} \in \mathcal{Z}}|f(\boldsymbol{z})|$. The set of bounded real functions on $\mathcal{Z}$ is $B(\mathcal{Z}):=\{f$ : $\mathcal{Z} \rightarrow \mathbb{R}$ such that $\left.\|f\|_{\infty}<\infty\right\}$.

- Let $\boldsymbol{a}=\left(a_{1}, \ldots, a_{d}\right)$ be a multi-index, where each $a_{i}$, $i=1,2, \ldots, d$, is a non-negative integer. Let $f: \mathcal{Z} \rightarrow \mathbb{R}$ be a real function on a $d$-dimensional set $\mathcal{Z} \subseteq \mathbb{R}^{d}$. We use $D^{a} f(z)$ to denote the partial derivative of $f$ w.r.t. the variable $z$ determined by the entries of $\boldsymbol{a}$, namely,

$$
D^{\boldsymbol{a}} f(\boldsymbol{z})=\frac{\partial^{a_{1}} \cdots \partial^{a_{d}} f}{\partial z_{1}^{a_{1}} \cdots \partial z_{d}^{a_{d}}}(\boldsymbol{z}) .
$$

The order of the derivative operator $D^{\boldsymbol{a}}$ is $|\boldsymbol{a}|=\sum_{i=1}^{d} a_{i}$.

- The minimum out of two scalar quantities, $a, b \in \mathbb{R}$, is denoted $a \wedge b$.

We make the following assumptions on the likelihood function $g_{t}$ and the predictive observation measure $\mu_{t}\left(d \boldsymbol{y}_{t}\right)=$ $p_{t}\left(\boldsymbol{y}_{t}\right) d \boldsymbol{y}_{t}$.

( $\mathfrak{L})$ For each $t \geq 1$, the function $g_{t}$ is positive and bounded, i.e., $g_{t}(\boldsymbol{y}, \boldsymbol{x})>0$ for any $(\boldsymbol{y}, \boldsymbol{x}) \in \mathcal{Y} \times \mathcal{X}$ and $\left\|g_{t}\right\|_{\infty}=$ $\sup _{(\boldsymbol{y}, \boldsymbol{x}) \in \boldsymbol{Y} \times \boldsymbol{X}}\left|g_{t}(\boldsymbol{y}, \boldsymbol{x})\right|<\infty$.

(D) For each $t \geq 1$, the function $g_{t}(\boldsymbol{y}, \boldsymbol{x})$ is differentiable with respect to $\boldsymbol{y}$, with bounded derivatives up to order $d_{y}$, hence $D^{\mathbf{1}} g_{t}(\boldsymbol{y}, \boldsymbol{x})=\frac{\partial^{d y} g_{t}}{\partial y_{1} \cdots \partial y_{d_{y}}}(\boldsymbol{y}, \boldsymbol{x})$ exists and

$$
\left\|D^{1} g_{t}\right\|_{\infty}=\sup _{(\boldsymbol{y}, \boldsymbol{x}) \in \mathcal{Y} \times \mathcal{X}}\left|D^{1} g_{t}(\boldsymbol{y}, \boldsymbol{x})\right|<\infty .
$$

(C) For any $0<\beta<1$ and any $p \geq 4$, the sequence of hypercubes

$$
\begin{aligned}
C_{M}:= & {\left[-\frac{M^{\frac{\beta}{p}}}{2},+\frac{M^{\frac{\beta}{p}}}{2}\right] \times \cdots } \\
& \times\left[-\frac{M^{\frac{\beta}{p}}}{2},+\frac{M^{\frac{\beta}{p}}}{2}\right] \subset \mathbb{R}^{d_{y}}
\end{aligned}
$$

satisfie the inequality $\mu_{t}\left(\overline{C_{M}}\right) \leq b M^{-\eta}$ for some constants $b>0$ and $\eta>0$ independent of $M$ (yet possibly dependent on $\beta$ and $p$ ), where $\overline{C_{M}}=\mathbb{R}^{d_{y}} \backslash C_{M}$ is the complement of $C_{M}$.

Remark 1: Assumptions ( $\mathfrak{L})$ and $(\mathfrak{D})$ refer to regularity conditions (differentiability and boundedness) that the likelihood function of the state space model should satisfy. Models of observations, for example, of the form $\boldsymbol{y}_{t}=f\left(\boldsymbol{x}_{t}\right)+\boldsymbol{u}_{t}$, where $f$ is a (possibly nonlinear) transformation of the state $\boldsymbol{x}_{t}$ and $\boldsymbol{u}_{t}$ is noise with some differentiable, exponential-type pdf(e.g., Gaussian or mixture-Gaussian), readily satisfy these assumptions. Typical two-sided heavy-tailed distributions, such as Student's $t$ distribution, also satisfy $(\mathfrak{L})$ and $(\mathfrak{D})$.

Remark 2: Assumption $(\mathfrak{C})$ states an explicit bound on the probability under the tails of the pdf $p_{t}\left(\boldsymbol{y}_{t}\right)=p\left(\boldsymbol{y}_{t} \mid \boldsymbol{y}_{1: t-1}\right)$. The bound is polynomial, namely

$$
\mu_{t}\left(\overline{C_{M}}\right)=1-\int_{-\frac{1}{2} M^{\frac{\beta}{p}}}^{\frac{1}{2} M^{\frac{\beta}{p}}} \cdots \int_{-\frac{1}{2} M^{\frac{\beta}{p}}}^{\frac{1}{2} M^{\frac{\beta}{p}}} p_{t}(\boldsymbol{y}) d \boldsymbol{y} \leq b M^{-\eta},
$$

and therefore immediately verified e.g., by all distributions of the exponential family as well as for many heavy-tailed distributions. For example, when $d_{y}=1$ (i.e., the observations are scalars), one can choose the constants $b$ and $\eta$ such that $b M^{-\eta}$ is an upper bound for the tails of the (heavy-tailed) Pareto, Weibull, Burr or Levy distributions.

It is actually possible to fin simple conditions on the conditional pdf of the observations, $g_{t}\left(\boldsymbol{y}_{t}, \boldsymbol{x}_{t}\right)$, that turn out sufficien for assumption $(\mathfrak{C})$ to hold true. Let us keep $d_{y}=1$, for simplicity, and assume that there exists a sequence of positive constants $\left\{c_{t}\right\}_{t \geq 1}$ such that $g_{t}\left(y_{t}, \boldsymbol{x}_{t}\right)$ has a polynomial upper bound itself, namely

$$
\sup _{\boldsymbol{x}_{t} \in \mathcal{X}} g_{t}\left(y_{t}, \boldsymbol{x}_{t}\right) \leq c_{t}\left|y_{t}\right|^{-(1+\epsilon)}
$$

for some $\epsilon>0$ and every $y_{t}$ such that $\left|y_{t}\right|>\frac{1}{2}$ (note that the smallest set in the sequence $C_{M}$ is $\left.C_{1}=\left[-\frac{1}{2}, \frac{1}{2}\right]\right)$. For probability distributions with infinit support and continuous with respect to the Lebesgue measure, the inequality (8) implies that the densities $g_{t}\left(y_{t}, \boldsymbol{x}_{t}\right)$ are integrable for every possible choice of $\boldsymbol{x}_{t} \in \mathcal{X}$. Then, the probability below the right tail of $p_{t}(y)$ is

$$
\begin{aligned}
\int_{\frac{1}{2} M^{\frac{\beta}{p}}}^{\infty} p_{t}(y) d y & =\int_{\frac{1}{2} M^{\frac{\beta}{p}}}^{\infty} \int_{\mathcal{X}} g_{t}(y, \boldsymbol{x}) \tilde{p}_{t}(\boldsymbol{x}) d \boldsymbol{x} d y \\
& \leq c_{t} \int_{\frac{1}{2} M^{\frac{\beta}{p}}}^{\infty} y^{-(1+\epsilon)} \int_{\mathcal{X}} \tilde{p}_{t}(\boldsymbol{x}) d \boldsymbol{x} d y
\end{aligned}
$$

where the inequality follows from the application of (8). Since $\tilde{p}_{t}(\boldsymbol{x})$ is a pdf, we have $\int_{\mathcal{X}} \tilde{p}_{t}(\boldsymbol{x}) d \boldsymbol{x}=1$ and some elementary calculations yield

$$
\begin{aligned}
\int_{\frac{1}{2} M^{\frac{\beta}{p}}}^{\infty} p_{t}(y) d y & \leq c_{t} \int_{\frac{1}{2} M^{\frac{\beta}{p}}}^{\infty} y^{-(1+\epsilon)} d y \\
& =c_{t}-\frac{y^{-\epsilon}}{\epsilon}{ }_{\frac{1}{2} M^{\frac{\beta}{p}}}^{\infty}=\frac{2^{\epsilon} c_{t}}{\epsilon} M^{-\frac{\epsilon \beta}{p}} .
\end{aligned}
$$

The same result is easily obtained for the left tail of $p_{t}(y)$, hence

$$
\begin{aligned}
\mu_{t}\left(\overline{C_{M}}\right) & =\int_{\frac{1}{2} M^{\frac{\beta}{p}}}^{\infty} p_{t}(y) d y+\int_{-\infty}^{-\frac{1}{2} M^{\frac{\beta}{p}}} p_{t}(y) d y \\
& \leq \frac{2^{1+\epsilon} c_{t}}{\epsilon} M^{-\frac{\epsilon \beta}{p}} .
\end{aligned}
$$

By comparing (10) and the inequality $\mu_{t}\left(\overline{C_{M}}\right) \leq b M^{-\eta}$, we readily see that we can choose $b=\frac{2^{1+\epsilon} c_{t}}{\epsilon}$ and $\eta=\frac{\epsilon \beta}{p}>0$ to uphold assumption $(\mathfrak{C})$. A similar derivation can be carried out when $d_{y}>1$.

Theorem 1: Assume that $(\mathfrak{L}),(\mathfrak{D})$ and $(\mathfrak{C})$ hold and the observations $\boldsymbol{y}_{1: t-1}$ are fi ed (and otherwise arbitrary). Then, for every $h \in B(\mathcal{Y})$ and any $\epsilon \in\left(0, \frac{1}{2}\right)$ there exists an a.s. finit r.v. $W_{t}^{\epsilon}$, independent of $M$, such that

$$
\left(h, \mu_{t}^{M}\right)-\left(h, \mu_{t}\right)^{\prime} \leq \frac{W_{t}^{\epsilon}}{M^{\left(\frac{1}{2}-\epsilon\right) \wedge \eta}} .
$$

In particular,

$$
\lim _{M \rightarrow \infty}\left(h, \mu_{t}^{M}\right)=\left(h, \mu_{t}\right) \quad \text { a.s. }
$$


See the Supplementary Material for a proof.

Note that the r.v. $W_{t}^{\epsilon}$ in the statement of Theorem 1 depends on the time instant $t$. It is possible to remove this dependence if the constants $b$ and $\eta$ in assumption ( $\mathfrak{C}$ ) are chosen to be independent of $t$ and we impose further constraints on the likelihood function and the Markov kernel of the state space model (similar to the sufficien conditions for uniform convergence in, e.g., [18] or [36]).

\section{ONLINE SElECtion OF THE NuMBER OF PARTIClES}

In the sequel we assume scalar observations, hence $d_{y}=1$ and $\boldsymbol{y}_{t}=y_{t}$ (while $d_{x} \geq 1$ is arbitrary). A discussion of how to proceed when $d_{y}>1$ is provided in Section IV-E.

Our goal is to evaluate the convergence of the BPF (namely, the accuracy of the approximation $\left.p_{t}^{M}\left(y_{t}\right)\right)$ in real time and, based on the convergence assessment, adapt the computational effort of the algorithm, i.e., the number of used particles $M$.

To that end, we run the BPF in the usual way with a light addition of computations. At each iteration we generate $K$ "fic titious observations", denoted $\tilde{y}_{t}^{(1)}, \ldots, \tilde{y}_{t}^{(K)}$, from the approximate predictive pdf $p_{t}^{M}\left(y_{t}\right)$. If the BPF is operating with a small enough level of error, then Theorem 1 states that these fictitiou observations come approximately from the same distribution as the acquired observation, i.e., $\mu_{t}^{M}\left(d y_{t}\right) \approx \mu_{t}\left(d y_{t}\right)$. In that case, as we explain in Subsection IV-B, a statistic $a_{t}^{K}$ can be constructed using $y_{t}, \tilde{y}_{t}^{(1)}, \ldots, \tilde{y}_{t}^{(K)}$, which necessarily has an (approximately) uniform distribution independently of the specifi form of the state-space model (1)-(3). By collecting a sequence of such statistics, say $a_{t-W+1}^{K}, \ldots, a_{t}^{K}$ for some window size $W$, one can easily test whether their empirical distribution is close to uniform using standard procedures. The better the approximation $\mu_{t}^{M} \approx \mu_{t}$ generated by the BPF, the better fi with the uniform distribution can be expected.

If $K<<M$ and $W$ is not too large, the cost of the added computations is negligible compared to the cost of running the BPF with $M$ particles and, as we numerically show in Section $\mathrm{V}$, the ability to adapt the number of particles online leads to a very significan reduction of the running times without compromising the estimation accuracy.

Below we describe the method, justify its theoretical validity and discuss its computational complexity as well as its extension to the case of multidimensional $\boldsymbol{y}_{t}$ 's.

\section{A. Generation of Fictitious Observations}

The proposed method demands at each time $t$ the generation of $K$ fictitiou observations (i.e., Monte Carlo samples), denoted $\left\{\tilde{y}_{t}^{(k)}\right\}_{k=1}^{K}$, from the approximate predictive observation pdf $p_{t}^{M}\left(y_{t}\right)=\frac{1}{M} \sum_{m=1}^{M} p\left(y_{t} \mid \overline{\boldsymbol{x}}_{t}^{(m)}\right)$. Since the latter density is a finit mixture, drawing from $p_{t}^{M}\left(y_{t}\right)$ is straightforward as long as the conditional density of the observations, $p\left(y_{t} \mid \boldsymbol{x}_{t}\right)$, is itself amenable to sampling. In order to generate $\tilde{y}_{t}^{(k)}$, it is enough to draw a sample $j^{(k)}$ from the discrete uniform distribution on $\{1,2, \ldots, M\}$ and then generate $\tilde{y}_{t}^{(k)} \sim p\left(y_{t} \mid \overline{\boldsymbol{x}}_{t}^{\left(j^{(k)}\right)}\right)$.

\section{B. Assessing Convergence Via Invariant Statistics}

For simplicity, let us assume firs that $p_{t}^{M}\left(y_{t}\right)=p_{t}\left(y_{t}\right)=$ $p\left(y_{t} \mid y_{1: t-1}\right)$, i.e., there is no approximation error and, therefore, the fictitiou observations $\left\{\tilde{y}_{t}^{(k)}\right\}_{k=1}^{K}$ have the same distribution as the true observation $y_{t}$. We defin the set $\mathcal{A}_{K, t}:=\left\{y \in\left\{\tilde{y}_{t}^{(k)}\right\}_{k=1}^{K}: y<y_{t}\right\}$ and the r.v. $A_{K, t}:=$ $\left|\mathcal{A}_{K, t}\right| \in\{0,1, \ldots, K\}$. Note that $\mathcal{A}_{K, t}$ is the set of fictitiou observations which are smaller than the actual one, while $A_{K, t}$ is the number of such observations. If we let $\mathbb{Q}_{K}$ denote the probability mass function (pmf) of $A_{K}$, it is not hard to show that $\mathbb{Q}_{K}$ is uniform independently of the value and distribution of $y_{t}$. This is rigorously given by the Proposition below.

Proposition 1: If $y_{t}, \tilde{y}_{t}^{(1)}, \ldots, \tilde{y}_{t}^{(K)}$ are i.i.d. samples from a common continuous (but otherwise arbitrary) probability distribution, then the pmf of the r.v. $A_{K, t}$ is

$$
\mathbb{Q}_{K}(n)=\frac{1}{K+1}, \quad n=0, \ldots, K .
$$

Proof: Since $y_{t}, \tilde{y}_{t}^{(1)}, \cdots, \tilde{y}_{t}^{(K)}$ are i.i.d., all possible orderings of the $K+1$ samples are a priori equally probable, and the value of the r.v. $A_{K, t}$ depends uniquely on the relative position of $y_{t}$ after the samples are sorted (e.g., if $y_{t}$ is the smallest sample, then $A_{K, t}=0$, if there is exactly one $\tilde{y}_{t}^{(i)}<y_{t}$ then $A_{K, t}=1$, etc.). There are $(K+1)$ ! different ways in which the samples $y_{t}, \tilde{y}_{t}^{(1)}, \cdots, \tilde{y}_{t}^{(K)}$ can be ordered, but $A_{K, t}$ can only take values from 0 to $K$. In particular, given the relative position of $y_{t}$, there are $K$ ! different ways in which the remaining samples $\tilde{y}_{t}^{(1)}, \cdots, \tilde{y}_{t}^{(K)}$ can be arranged. Therefore, $\mathbb{Q}_{K}\left(A_{K}=n\right)=\frac{K !}{(K+1) !}=\frac{1}{K+1}$ for every $n \in$ $\{0,1, \ldots, K\}$.

For the case of interest in this paper, the r.v.'s $y_{t}$, $\tilde{y}_{t}^{(1)}, \ldots, \tilde{y}_{t}^{(K)}$ (the actual and fictitiou observations) have a common probability distribution given by the measure $\mu_{t}$ and are generated independently. For the class of state space models described in Section II, and the explicit assumptions in Section III, the measure $\mu_{t}$ is absolutely continuous w.r.t. the Lebesgue measure (with associated density $p_{t}(y)$ ) and, therefore, $y_{t}, \tilde{y}_{t}^{(1)}, \ldots, \tilde{y}_{t}^{(K)}$ are indeed continuous r.v.'s and the assumptions of Proposition 1 are met. Moreover, it can also be proved that the variables in the sequence $A_{K, t}$ are independent.

Proposition 2: If the r.v.'s $y_{t}, \tilde{y}_{t}^{(1)}, \ldots, \tilde{y}_{t}^{(K)}$ are i.i.d. with common pdf $p_{t}(y)$, then the r.v.'s in the sequence $\left\{A_{K, t}\right\}_{t \geq 1}$ are independent.

See Appendix A for a proof.

In practice, $p_{t}^{M}\left(y_{t}\right)$ is just an approximation of the predictive observation pdf $p_{t}\left(y_{t}\right)$ and, therefore, the actual and ficti tious observations are not i.i.d. However, under the assumptions of Theorem 1, the a.s. convergence of the approximate measure $\mu_{t}^{M}\left(d y_{t}\right)=p_{t}^{M}\left(y_{t}\right) d y_{t}$ enables us to obtain an "approximate version" of the uniform distribution in Proposition 1, with the error vanishing as $M \rightarrow \infty$. To be specific we introduce the set $\mathcal{A}_{K, M, t}:=\left\{y \in\left\{\tilde{y}_{t}^{(k)}\right\}_{k=1}^{K}: y<y_{t}\right\}$, which depends on $M$ because of the mismatch between $p_{t}^{M}\left(y_{t}\right)$ and $p_{t}\left(y_{t}\right)$, and the 
TABLE I

PARAMETERS OF THE ALGORITHM

- $M_{0}$, initial number of particles

- $M_{\min }$, minimum number of particles

- $M_{\max }$, maximum number of particles

- $K$, number of fictitious samples per iteration

- $W$, window length

- $p_{\ell}$, lower significance level of $\mathrm{p}$-values

- $p_{h}$, higher significance level of $\mathrm{p}$-values

- $f_{\text {up }}(\cdot)$, rule for increasing $M$

- $f_{\text {down }}(\cdot)$, rule for decreasing $M$

associated r.v. $A_{K, M, t}=\left|\mathcal{A}_{K, M, t}\right|$ with pmf $\mathbb{Q}_{K, M, t}$. We have the following convergence result for $\mathbb{Q}_{K, M, t}$.

Theorem 2: Let $y_{t}$ be a sample from $p_{t}\left(y_{t}\right)$ and let $\left\{\tilde{y}_{t}^{(k)}\right\}_{k=1}^{K}$ be i.i.d. samples from $p_{t}^{M}\left(y_{t}\right)$. If the observations $y_{1: t-1}$ are fi ed and Assumptions $(\mathfrak{L}),(\mathfrak{D})$ and $(\mathfrak{C})$ hold, then there exists a sequence of non-negative r.v.'s $\left\{\varepsilon_{t}^{M}\right\}_{M \in \mathbb{N}}$ such that $\lim _{M \rightarrow \infty} \varepsilon_{t}^{M}=0$ a.s. and

$$
\frac{1}{K+1}-\varepsilon_{t}^{M} \leq \mathbb{Q}_{K, M, t}(n) \leq \frac{1}{K+1}+\varepsilon_{t}^{M} .
$$

In particular, $\lim _{M \rightarrow \infty} \mathbb{Q}_{K, M, t}(n)=\mathbb{Q}_{K}(n)=\frac{1}{K+1}$ a.s.

See Appendix B for a proof. Proposition 1 states that the statistic $A_{K, t}$ is distribution-invariant, since $\mathbb{Q}_{K}(n)=\frac{1}{K+1}$ independently of $t$ and the state space model. Similarly, Theorem 2 implies that the statistic $A_{K, M, t}$ is asymptotically distribution-invariant (independently of $t$ and the model) since $\mathbb{Q}_{K, M, t}(n) \rightarrow \frac{1}{K+1}$ when $M \rightarrow \infty$, as the BPF converges. ${ }^{3}$

\section{Algorithm with Adaptive Number of Particles}

We propose an algorithm that dynamically adjusts the number of particles of the filte based on the transformed r.v. $A_{K, M, t}$. Table II summarizes the proposed algorithm, that is embedded into a standard BPF (see Section II-B) but can be applied to virtually any other particle filte in a straightforward manner. The parameters of the algorithm are shown in Table I.

The BPF is initialized in Step 1(a) with $M_{0}$ initial particles. At each recursion, in Step 2(a), the filtere distribution of the current state is approximated. In Step 2(b), $K$ fictitiou observations $\left\{\tilde{y}_{t}^{(k)}\right\}_{k=1}^{K}$ are drawn and the statistic $A_{K, M, t}=a_{K, M, t}$ is computed. In Step 2(c), once a set of $W$ consecutive statistics have been acquired, $\mathcal{S}_{t}=$ $\left\{a_{K, M, t-W+1}, a_{K, M, t-W+2}, \ldots, a_{K, M, t-1}, a_{K, M, t}\right\}$, a statistical test is performed for checking whether $\mathcal{S}_{t}$ is a sequence of samples from the uniform pmf given by Eq. (11).

There are several approaches that can be used to exploit the information contained in $\mathcal{S}_{t}$. Here we perform a Pearson's chisquared test [37], where the $\chi_{t}^{2}$ statistic is computed according to Eq. (13) (see Table II). Then, a p-value $p_{K, t}^{*}$ for testing the hypothesis that the empirical distribution of $\mathcal{S}_{t}$ is uniform is computed. The value $p_{K, t}^{*}$ is obtained by comparing the $\chi_{t}^{2}$ statistic

\footnotetext{
${ }^{3}$ Specificall note that, under assumptions $(\mathfrak{L}),(\mathfrak{D})$ and $(\mathfrak{C})$, the convergence of the continuous random measure $\mu_{t}^{M}$ computed via the BPF (which is suffi cient to obtain (12); see Appendix B) is guaranteed by Theorem 1.
}

TABLE II

ALGORITHM FOR ADAPTING THE NUMBER OF PARTICLES

1) [Initialization]

a) Initialize the particles and the weights of the filter as

$$
\begin{aligned}
\boldsymbol{x}_{0}^{(m)} & \sim p\left(\boldsymbol{x}_{0}\right), & & m=1, \ldots, M_{0}, \\
w_{0}^{(m)} & =1 / M_{0}, & & m=1, \ldots, M_{0},
\end{aligned}
$$

and set $n=1$.

2) [For $t=1: T]$

a) Bootstrap particle filter:

- Resample $M_{n}$ samples of $\overline{\boldsymbol{x}}_{t-1}^{(m)}$ with weights $w_{t-1}^{(m)}$ to obtain $\boldsymbol{x}_{t-1}^{(m)}$.

- Propagate $\overline{\boldsymbol{x}}_{t}^{(m)} \sim p\left(\boldsymbol{x}_{t} \mid \boldsymbol{x}_{t-1}^{(m)}\right), \quad m=1, \ldots, M_{n}$.

- Compute the non-normalized weights $\bar{w}_{t}^{(m)}=p\left(y_{t} \mid \overline{\boldsymbol{x}}_{t}^{(m)}\right)$, $m=1, \ldots, M_{n}$

- Normalize the weights $\bar{w}_{t}^{(m)}$ to obtain $w_{t}^{(m)}, \quad m=$ $1, \ldots, M_{n}$

b) Fictitious observations:

- Draw $\tilde{y}_{t}^{(k)} \sim p^{M}\left(y_{t} \mid y_{t-1}\right), k=1, \ldots, K$.

- Compute $a_{K, M, t}=A_{K, M, t}$, i.e., the position of $y_{t}$ within the set of ordered fictitious observations $\left\{\tilde{y}_{t}^{(k)}\right\}_{k=1}^{K}$

c) If $t=n W$, (assessment of convergence):

- Compute the $\chi_{t}^{2}$ statistic over the empirical distribution of $\mathcal{S}_{t}=\left\{a_{K, M, t}, a_{K, M, t-1}, \ldots, a_{K, M, t-W+1}\right\}$ as

$$
\chi_{t}^{2}=\sum_{j=0}^{K} \frac{\left(O_{j}-E_{j}\right)^{2}}{E_{j}},
$$

where $O_{j}$ is the frequency of the observations in the window being in the $j$ th relative position, i.e., $O_{j}=\mid a_{K, M, \tau} \in$ $\mathcal{S}_{t}: a_{K, M, \tau}=j \mid$, and $E_{j}$ is the expected frequency under the null hypothesis, i.e., $E_{j}=W \cdot \mathbb{Q}_{K}(j)=\frac{W}{K+1}$ (see Eq. (11)).

- Calculate the p-value $p_{K, t}^{*}$ by comparing the statistic $\chi_{t}^{2}$ to the $\chi^{2}$-distribution with $K$ degrees of freedom.

- If $p_{K, t}^{*} \leq p_{\ell}$ increase $M_{n}=\min \left\{f_{\text {up }}\left(M_{n-1}\right), M_{\max }\right\}$.

- Else, if $p_{K, t}^{*} \geq p_{h}$, decrease $M_{n}=\max \left\{f_{\text {down }}\left(M_{n-1}\right), M_{\min }\right\}$

- Else, $M_{n}=M_{n-1}$

- Set $n=n+1$.

d) If $t<W n$, set $t=t+1$ and go to 2 . Otherwise, end.

with the $\chi^{2}$ distribution with $K$ degrees of freedom. Intuitively, a large $p_{K, t}^{*}$ suggests a good match of the sequence $\mathcal{S}_{t}$ with an i.i.d. sample from the uniform distribution on $\{0,1, \ldots, K\}$, while a small $p_{K, t}^{*}$ indicates a mismatch. Therefore, the $\mathrm{p}$-value $p_{K, t}^{*}$ is compared with two different significanc levels: a low threshold $p_{\ell}$ and a high threshold $p_{h}$. If $p_{K, t}^{*} \leq p_{\ell}$, the number of particles is increased according to the rule $M_{t}=f_{\text {up }}\left(M_{t-1}\right)$ whereas, if $p_{K, t}^{*} \geq p_{h}$, the number of particles is decreased according to the rule $M_{t}=f_{\text {down }}\left(M_{t-1}\right)$. When $p_{\ell}<p_{K, t}^{*}<p_{h}$, the number of particles remains fi ed. These two significanc levels allow the practitioner to select the operation range by considering a performance-to-computational-cost tradeoff. Note that we set $M_{\min }$ and $M_{\max }$, maximum and minimum values for the number of particles, respectively.

A large window $W$ yields a more accurate convergence assessment but increases the latency (or decreases the 
responsiveness) of the algorithm. If the algorithm must be run online, this latency can be critical for detecting a malfunction of the filte and adapting consequently the number of particles. Therefore there is a tradeoff between the accuracy of the convergence assessment procedure and latency of the algorithm.

\section{Computational Cost}

Compared to the BPF, the additional computational cost of the method is mainly driven by the generation of the $K$ fictitiou observations at each iteration as shown in Subsection IV-A. The generation of these fictitiou observations is a two-step procedure, where in the firs step, we draw $K$ discrete indices, say $j_{1}, \ldots, j_{K}$, from the set $\left\{1, \ldots, M_{n}\right\}$ with uniform probabilities, and in the second step, we draw $K$ samples from $p\left(y_{t} \mid \overline{\boldsymbol{x}}_{t}^{\left(j_{1}\right)}\right), \ldots, p\left(y_{t} \mid \overline{\boldsymbol{x}}_{t}^{\left(j_{K}\right)}\right)$, respectively.

In the proposed algorithm, a Pearson's $\chi^{2}$ test is performed with a sequence $\mathcal{S}_{t}$ of $W$ samples, that is, it is carried out only once every $W$ consecutive time steps. Therefore, the computational cost will depend on the parameters $K$ and $W$. We will show in Section $\mathrm{V}$ that the algorithm can work very well with a low number of fictitiou observations, which imposes a very light extra computational load.

\section{E. Multidimensional Observations}

Through this section, we have assumed scalar observations. In the multidimensional case, with $\boldsymbol{y}_{t}=\left[y_{1, t}, \ldots, y_{d_{y}, t}\right]^{\top}$, the same assessment scheme can be applied over each marginal $p\left(y_{i, t} \mid \boldsymbol{y}_{1: t-1}\right)$ of the predictive observation pdf. Theoretical guarantees readily follow from the convergence of the marginal measures $\mu_{i, t}^{M}\left(d y_{i, t}\right)=p^{M}\left(y_{i, t} \mid \boldsymbol{y}_{1: t-1}\right) d y_{i, t}$ under the same assumptions as the joint measure $\mu_{t}^{M}$ (see the Supplementary Material).

The algorithm proposed in Section IV-C can be extended to the case with multidimensional observations. One way of doing it is by performing an independent assessment for each marginal pdf $p\left(y_{i, t} \mid \boldsymbol{y}_{1: t-1}\right)$. As a result, $d_{y}$ p-values $p_{K, t, i}^{*}$, with $i=$ $1, \ldots, d_{y}$, become available for deciding whether to increase, decrease or keep fi ed the number of particles. A conservative approach is to increase the number of particles whenever at least one $\mathrm{p}$-value $p_{K, t, i}^{*}$ is below the threshold $p_{\ell}$. Note that the complexity of this approach grows with the dimension of the observations.

Finally, note that the convergence of the marginals does not imply the convergence of the joint approximation $\mu_{t}^{M}$. However, it can be reasonably expected that when all the marginals are approximated well over a period of time, the joint distribution is accurately approximated as well.

\section{NumericAl EXAMPLE}

\section{A. The Three-dimensional Lorenz System}

1) Model Description: In this section we show computer simulation results that demonstrate the performance of the proposed method. We consider the problem of tracking the state of a three-dimensional Lorenz 63 system [38] with additive dynamical noise, partial observations and additive measurement noise [39]. Namely, we consider a three-dimensional stochastic process $\{\boldsymbol{X}(s)\}_{s \in(0, \infty)}$ taking values on $\mathbb{R}^{3}$, whose dynamics are described by the system of stochastic differential equations

$$
\begin{aligned}
& d X_{1}=-s\left(X_{1}-Y_{1}\right)+d W_{1} \\
& d X_{2}=r X_{1}-X_{2}-X_{1} X_{3}+d W_{2} \\
& d X_{3}=X_{1} X_{2}-b X_{3}+d W_{3}
\end{aligned}
$$

where $\left\{W_{i}(s)\right\}_{s \in(0, \infty)}, \quad i=1,2,3$, are independent onedimensional Wiener processes and

$$
(\mathrm{s}, \mathrm{r}, \mathrm{b})=\left(10,28, \frac{8}{3}\right.
$$

are static model parameters broadly used in the literature since they lead to a chaotic behavior [38]. Here we use a discrete-time version of the latter system using an Euler-Maruyama scheme with integration step $\Delta=10^{-3}$, which yields the model

$$
\begin{aligned}
X_{1, n}= & X_{1, n-1}-\Delta s\left(X_{1, n-1}-X_{2, n-1}\right)+\sqrt{\Delta} U_{1, n} \\
X_{2, n}= & X_{2, n-1}+\Delta\left(r X_{1, n-1}-X_{2, n-1}-X_{1, n-1} X_{3, n-1}\right) \\
& +\sqrt{\Delta} U_{2, n} \\
X_{3, n}= & X_{3, n-1}+\Delta\left(X_{1, n-1} X_{2, n-1}-b X_{3, n-1}\right) \\
& +\sqrt{\Delta} U_{3, n}
\end{aligned}
$$

where $\left\{U_{i, n}\right\}_{n=0,1, \ldots}, i=1,2,3$, are independent sequences of i.i.d. normal random variables with zero mean and unit variance. The system (14)-(16) is partially observed every 200 discretetime steps. Specificall, we collect a sequence of scalar observations $\left\{Y_{t}\right\}_{t=1,2, \ldots}$, of the form

$$
Y_{t}=X_{1,200 t}+V_{t}
$$

where the observation noise $\left\{V_{t}\right\}_{t=1,2, \ldots}$ is a sequence of i.i.d. normal random variables with zero mean and variance $\sigma^{2}=\frac{1}{2}$.

Let $\boldsymbol{X}_{n}=\left(X_{1, n}, X_{2, n}, X_{3, n}\right) \in \mathbb{R}^{3}$ be the state vector. The dynamic model given by Eqs. (14)-(16) define the transition kernel $p\left(\boldsymbol{x}_{n} \mid \boldsymbol{x}_{n-1}\right)$ and the observation model of Eq. (17) is the likelihood function

$$
\left.p\left(y_{t} \mid x_{1,200 t}\right) \propto \exp -\frac{1}{2 \sigma^{2}}\left(y_{t}-x_{1,200 t}\right)^{2}\right\} .
$$

The goal is on tracking the sequence of joint posterior probability measures $\pi_{t}, t=1,2, \ldots$, for $\left\{\hat{\boldsymbol{X}}_{t}\right\}_{t=1, \ldots}$, where $\hat{\boldsymbol{X}}_{t}=$ $\boldsymbol{X}_{200 t}$. Note that one can draw a sample $\hat{\boldsymbol{X}}_{t}=\hat{\boldsymbol{x}}_{t}$ conditional on $\hat{\boldsymbol{X}}_{t-1}=\hat{\boldsymbol{x}}_{t-1}$ by successively simulating

$$
\tilde{\boldsymbol{x}}_{n} \sim p\left(\boldsymbol{x}_{n} \mid \tilde{\boldsymbol{x}}_{n-1}\right), \quad n=200(t-1)+1, \ldots, 200 t,
$$

where $\tilde{\boldsymbol{x}}_{200(t-1)}=\hat{\boldsymbol{x}}_{t-1}$ and $\hat{\boldsymbol{x}}_{t}=\tilde{\boldsymbol{x}}_{200 t}$. The prior measure for the state variables is normal, namely

$$
\boldsymbol{X}_{0} \sim \mathcal{N}\left(\boldsymbol{x}_{*}, v_{0}^{2} \mathcal{I}_{3}\right)
$$

where $\boldsymbol{x}_{*}=(-5.9165 ;-5.5233 ; 24.5723)$ is the mean and $v_{0}^{2} \mathcal{I}_{3}$ is the covariance matrix of $\boldsymbol{X}_{0}$, with $v_{0}^{2}=10$ and $\mathcal{I}_{3}$ being the three-dimensional identity matrix. 
TABLE III

Lorenz Model (SECtion V-A): $\Delta=10^{-3}, T_{o b s}=200 \Delta, \sigma^{2}=0.5$. AlgORIthm Details: $W=20, K=7, M_{\mathrm{MAX}}=2^{15}, M_{\mathrm{MIN}}=2^{7}$. MSE IN THE Approximation of the Posterior Mean, Averaged Number of Particles $\bar{M}$, AVEraged p-Value, and Averaged Hellinger Distance

\begin{tabular}{|l|c|c|c|c|c|c|}
\hline$\left[p_{l}-p_{h}\right]$ & Fixed $M=2^{15}$ & {$[0.4-0.8]$} & {$[0.35-0.7]$} & {$[0.3-0.7]$} & {$[0.25-0.65]$} & {$[0.2-0.6]$} \\
\hline MSE & 1.5193 & 1.5234 & 1.5240 & 1.5287 & 3.7552 & 4.6540 \\
\hline$M$ & 32768 & 24951 & 14840 & 8729 & 2197 & 451 \\
\hline p-val & 0.5108 & 0.5089 & 0.4902 & 0.4815 & 0.4872 & 0.4785 \\
\hline Hell. distance & 0.2312 & 0.2355 & 0.2493 & 0.2462 & 0.2476 & 0.2521 \\
\hline exec. time (s) & 6201 & 5617 & 3014 & 1532 & 131 & 67 \\
\hline time ratio & 1 & 1.10 & 2.1 & 4.05 & 47.43 & 92.36 \\
\hline
\end{tabular}

2) Simulation Setup: With this example, we aim at showing how the proposed algorithm allows to operate the particle filte with a prescribed performance-to-computational-budget tradeoff. With this purpose, we applied a standard BPF for tracking the sequence of posterior probability measures of the system (14)-(16) generated by the three-dimensional Lorenz model described above. We generated a sequence of $T=2000$ synthetic observations, $\left\{y_{t} ; t=1, \ldots, 2000\right\}$, spread over an interval of 400 seconds (in continuous time), corresponding to $4 \times 10^{5}$ discrete time steps in the Euler-Maruyama scheme (hence, one observation every 200 steps). Since the time scale of the discrete time approximation of Eqs. (14)-(16) is $n=200 t$, a resampling step is taken every 200 steps of the underlying discrete-time system.

We started running the PF with a sufficientl large number of particles, namely $M=5000$, and then let the proposed algorithm decrease the number of particles to attain a prescribed point in the performance-to-computation-cost range. This point is controlled by the operation range of the p-value, which is in turn driven by the pair of signifi cance levels $\left[p_{\ell}-p_{h}\right]$. We tested the algorithm for different ranges of p-values, namely, $p_{\ell} \in\{0.5,0.4,0.3,0.2,0.1,0.05\}$ and $p_{h} \in\{0.9,0.8,0.7,0.6,0.5,0.4,0.3,0.2,0.1\}$. When the $p-$ value is below $p_{\ell}$, the algorithm doubles the number of particles $M_{n+1}=f_{\text {up }}\left(M_{n}\right)=2 M_{n}$, and when the p-value is over $p_{h}$, the number of particles is halved, $M_{n+1}=f_{\text {down }}\left(M_{n}\right)=M_{n} / 2$. We used $K=7$ fictitiou observations and a window of size $W=20$.

In order to assess the approximation errors, we computed the empirical MSEs of the approximation of the posterior mean, $E\left[\hat{\boldsymbol{X}}_{t} \mid Y_{1: t}=y_{1: t}\right]$, by averaging the MSEs for the whole sequences. Note that, since the actual expectation cannot be computed in closed form for this system, we used the true underlying sequence $\left\{\boldsymbol{X}_{200 t}\right\}_{t=1,2, \ldots}$ as the ground truth.

3) Numerical Results: Table III shows results of the MSE of the approximation of the posterior mean, the average number of particles

$$
\bar{M}=\frac{2}{T} \sum_{k=\frac{T}{2}+1}^{T} M_{k},
$$

the p-values of the $\chi^{2}$ test, and the Hellinger distance [40] between the empirical distribution of $\mathcal{S}_{t}$ and the uniform distribution. They were obtained by averaging over 100 runs and averaging over time for each run. The initial number of particles $M_{0}=2^{15}$, and the minimum and maximum number of particles
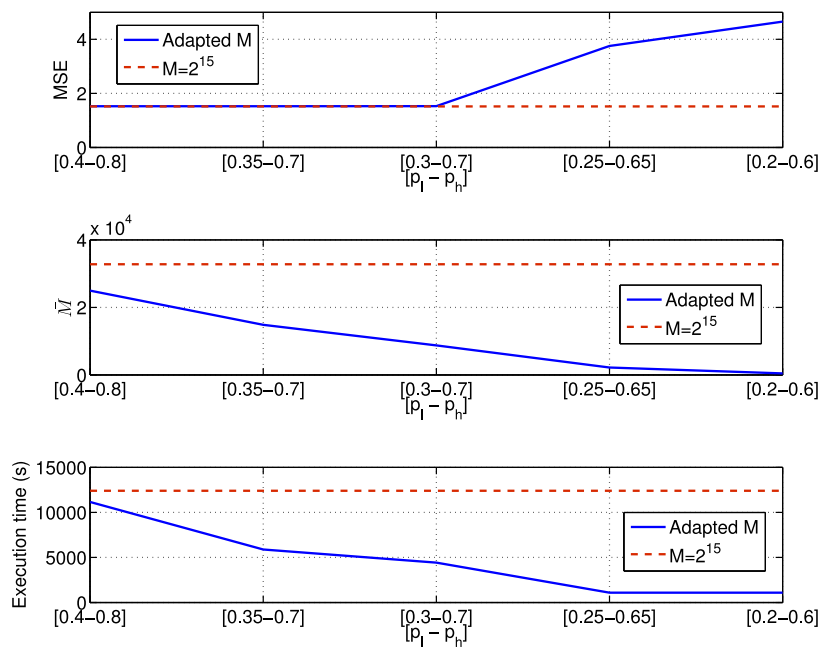

Fig. 1. Lorenz Model (Section V-A). MSE, number of particles $M$ and execution time for different pairs of significanc levels $\left[p_{\ell}-p_{h}\right]$ in solid blue line, and with a fi ed number of particles $M=2^{15}$ in dashed red line.

are $M_{\min }=2^{5}$ and $M_{\max }=2^{15}$, respectively. The firs half of time steps were discarded for obtaining the displayed results in order to test the behavior of the algorithm for different sets of parameters (see Eq. (18)). Regarding the relation between the MSE and $\bar{M}$ and the p-values, it can be seen that selecting a high operation range yields good performance (low MSE) at the cost of using a large number of particles (high $\bar{M}$ ). When we decrease the range of p-values, the algorithm decreases the number of particles, increasing also the approximation error. Table III shows that this conclusion holds for any pair of $\left[p_{\ell}-p_{h}\right]$.

Figure 1 shows the MSE, the number of particles $\bar{M}$, and the execution time for the different operation ranges (solid blue line) compared to the particle filte with a fi ed number of particles $M=2^{15}$ (dashed red line). It can be seen that with a moderate operation range $\left(\left[p_{\ell}-p_{h}\right]=[0.3-0.7]\right)$, the algorithm can perform (in terms of MSE) similarly to the case with fi ed $M$, while reducing the execution time approximately by a factor of four. The execution time can be further reduced by decreasing the operation range, although this worsens the performance.

Figure 2 displays the evolution of the number of particles over time (averaged over 100 runs) for $\left[p_{\ell}-p_{h}\right]=[0.3-0.7]$ both when $M_{0}=5000$ and $M_{0}=10$. In this case, the minimum and maximum number of particles are $M_{\min }=10$ and $M_{\max }=$ 5000 , respectively. We see that, after some time, the number of particles adjusted by the algorithm does not depend on $M_{0}$. 


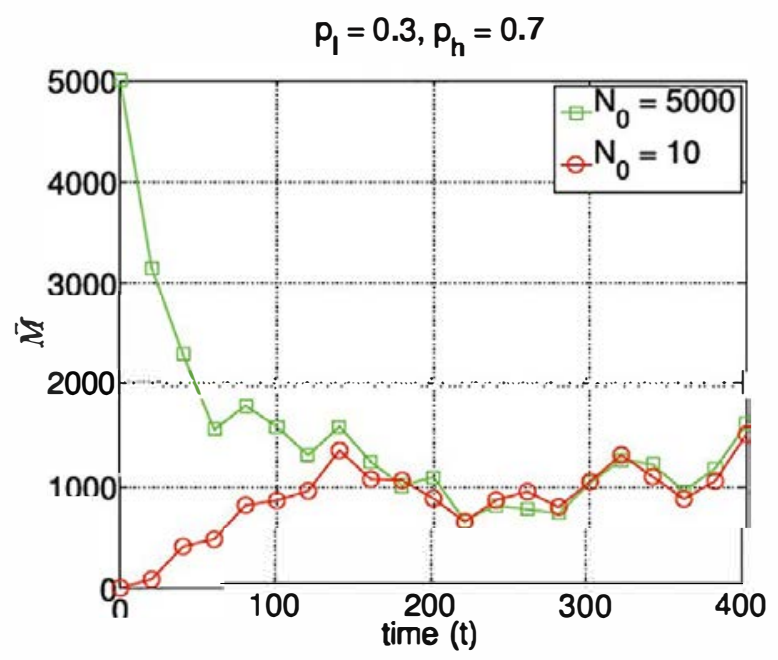

Fig. 2. Lorenz Model (Section V-A). Evolution of the number of particles adapted by the proposed algorithm when the initial number of particles $M_{0} \in$ $\{10,5000\}$. The significance levels were set to $p_{\ell}=0.3$ and $p_{h}=0.7$.

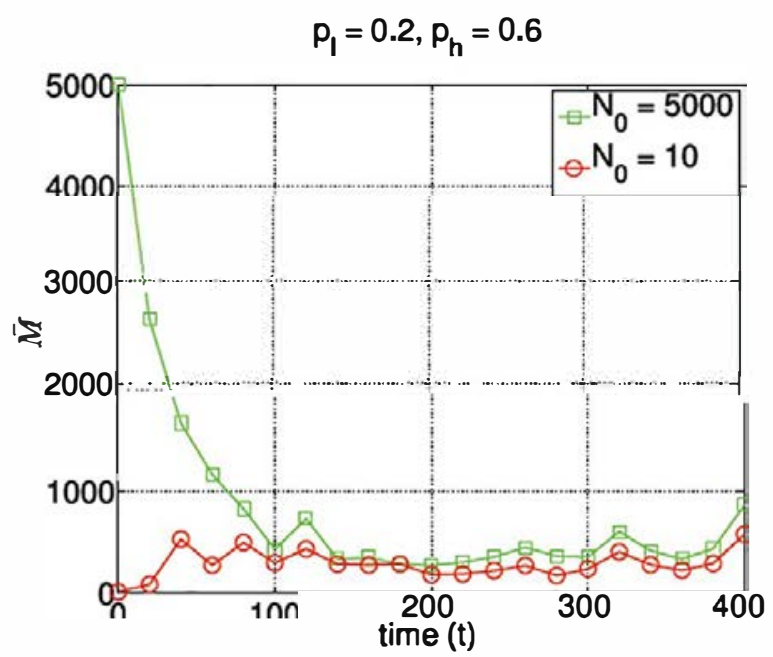

Fig. 3. Lorenz Model (Section V-A). Evolution of the number of particles adapted by the proposed algorithm when the initial number of particles $M_{0} \in$ $\{10,5000\}$. The significance levels were set to $p_{\ell}=0.2$ and $p_{h}=0.6$.

Figure 3 shows the same hehavior for $\left[p_{\ell}-p_{h}\right]=[0.2-0.6]$. After some time, the filter uses less particles than the filter with results in Fig. 2 because the selected range of thresholds employs smaller p-values.

Figure 4 shows histograms of averaged MSE and $M$ for simulations performed with two different sets of thresholds: $\left[p_{\ell}-p_{h}\right]=[0.3-0.5]$ and $\left[p_{\ell}-p_{h}\right]=[0.5-0.7]$. In both cases, the initial number of particles is $M_{0}=5000$. It can be seen that a more demanding pair of thresholds $\left(\left[p_{\ell}-p_{h}\right]=\right.$ $[0.5-0.7])$ leads to better performance and a larger average number of particles. This behavior can also be seen in Figure 5, where the MSE w.r.t. the number of particles is displayed for three different sets of thresholds. Note that a filter with a 100 relaxed set of thresholds $\left(\left[p_{\ell}-p_{h}\right]=[0.05-0.4]\right)$ uses very few particles but obtains a poor performance, while a filter with the most stringent set of thresholds $\left(\left[p_{\ell}-p_{h}\right]=[0.5-0.9]\right)$
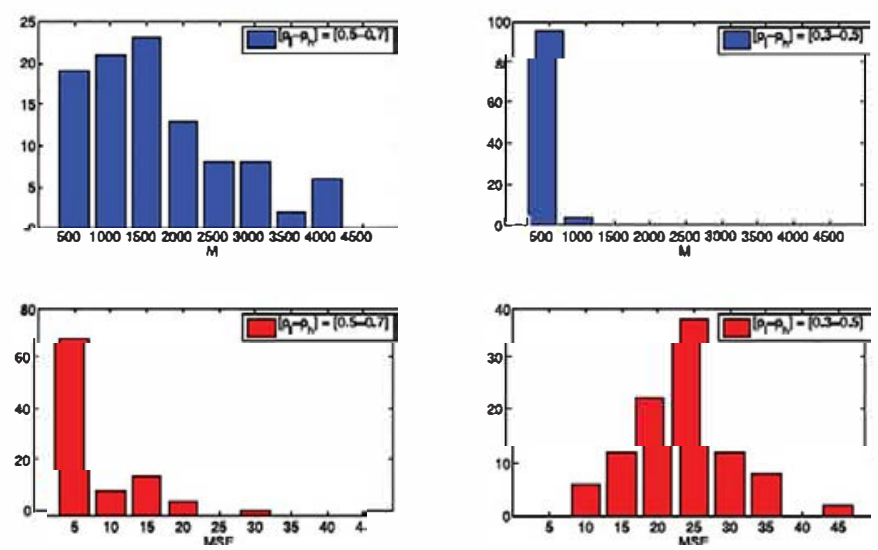

Fig. 4. Lorenz Model (Section V-A). Histograms of averaged MSE and M with $\left[p_{\ell}-p_{h}\right]=[0.3-0.5]$ and $\left[p_{\ell}-p_{h}\right]=[0.5-0.7]$. In both cases, the initial number of particles $M_{0}=5000$.

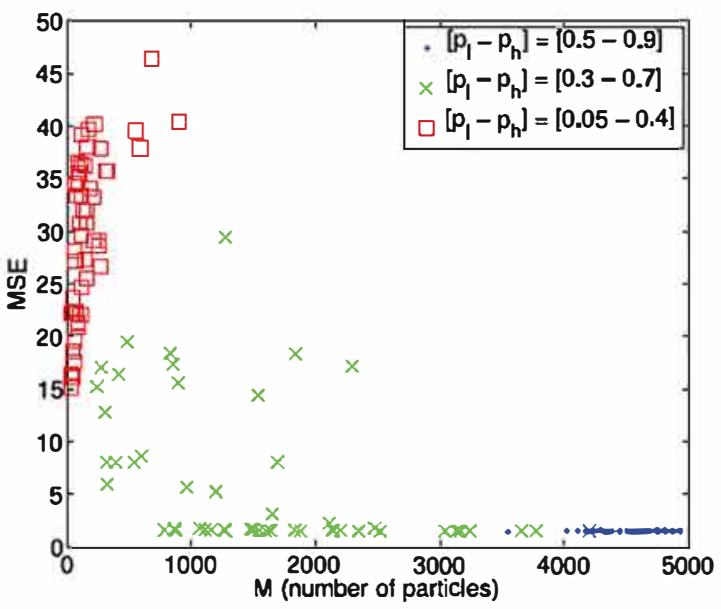

Fig. 5. Lorenz Model (Section V-A). MSE w.r.t. the averaged number of particles $M$ for runs with different sets of thresholds.

consistently yields a low MSE, at the expense of using a larger number of particles.

The numerical results have been computed in a Matlab environment on a computer with an Intel Core i5 processor (2.7 GHz clock frequency) and $12 \mathrm{~GB}$ of RAM.

4) Multidimensional Observations: Now we consider the case where we have observations also related to the second dimension of the hidden state. In particular, and following the notation of the previous experiment, we collect a sequence of hi-dimensional observations $\left\{Y_{t}\right\}_{t=1,2, \ldots}$ with components

$$
\begin{aligned}
& Y_{1, t}=X_{1,400 t}+V_{1, t}, \\
& Y_{2, t}=X_{2,400 t}+V_{2, t},
\end{aligned}
$$

where the observation noises $\left\{V_{1, t}\right\}_{t=1,2, \ldots}$ and $\left\{V_{2, t}\right\}_{t=1,2, \ldots}$ are two sequences of i.i.d. normal random variables with zero mean and variance $\sigma^{2}=\frac{1}{2}$. Note that now the state is observed every 400 discrete-time steps in order to make the system more difficult to be tracked.

The implemented algorithm is an extension of the unidimensional case, as suggested in Section IV-E. In particular, we 
TABLE IV

Outputs of the Particle Filter with Adaptive $M$ For the Lorenz Model (Section V-A) with Parameters $\Delta=10^{-3}, T_{o} b=400 \Delta, \sigma^{2}=0.5$ and

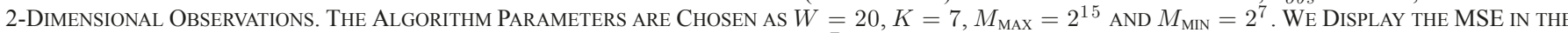
Approximation of the Posterior Mean, the Averaged Number of Particles $\bar{M}$, Averaged p-Value (Over Both Dimensions), and the Running Time

\begin{tabular}{|l|c|c|c|c|c|c|c|}
\hline$\left[p_{l}-p_{h}\right]$ & {$[0.4-0.8]$} & {$[0.3-0.7]$} & {$[0.3-0.65]$} & {$[0.25-0.65]$} & {$[0.2-0.6]$} & {$[0.15-0.55]$} & {$[0.1-0.5]$} \\
\hline MSE & 2.7151 & 2.7131 & 2.8351 & 3.8862 & 4.0814 & 5.4015 & 7.0323 \\
\hline$M$ & 26175 & 19652 & 15788 & 7761 & 3858 & 539 & 203 \\
\hline p-val & 0.5020 & 0.4953 & 0.4858 & 0.4906 & 0.4914 & 0.4820 & 0.4869 \\
\hline exec. time (s) & 2937.9851 & 2120.0787 & 1744.3426 & 772.2125 & 373.6780 & 73.1735 & 38.3487 \\
\hline
\end{tabular}
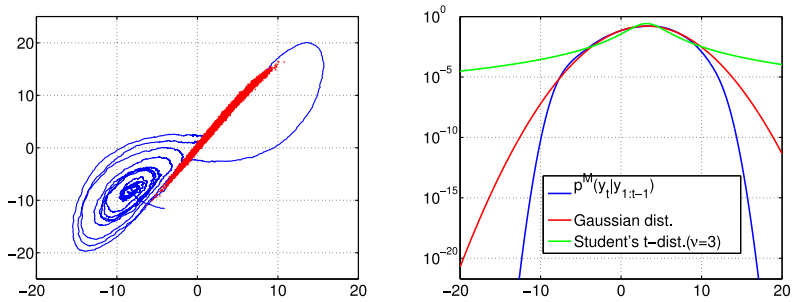

Fig. 6. Approximated observation predictive pdf $p^{M}\left(y_{t} \mid y_{1: t-1}\right)$, Gaussian distribution, and Student's t-distribution $(\nu=3)$ in log-scale for the stochastic Lorenz 63 example with $M=2^{14}$ particles. All distributions have the same mean and variance.

perform the assessment over the marginals $p\left(y_{i, t} \mid \boldsymbol{y}_{1: t-1}\right)$, with $i=1,2$, and then, with both $\mathrm{p}$-values, we adapt the number of particles as follows: if at least one of the marginals requires more particles, we increase the number of particles; if both marginals indicate no need for change of the number of particles, we keep it fi ed; otherwise, we decrease the number.

Table IV shows the MSE in the approximation of the posterior mean, averaged number of particles $\bar{M}$, averaged p-value (over both dimensions), and the running time. Note that we can extract similar conclusions as in the case with scalar observations.

5) Discussion: The assumption $(\mathfrak{C})$ of Section III states that the tails of the pdf $p\left(\boldsymbol{y}_{t} \mid \boldsymbol{y}_{1: t-1}\right)$ should not be too heavy. Nevertheless, we have shown that the constraint is rather weak, since it is satisfie for all exponential-type distributions as well as for many heavy-tailed distributions. In practice, $p\left(\boldsymbol{y}_{t} \mid \boldsymbol{y}_{1: t-1}\right)$ cannot be characterized for most models in a closed form. Here we show the particle approximation of the observation predictive pdf $p^{M}\left(\boldsymbol{y}_{t} \mid \boldsymbol{y}_{1: t-1}\right)$ in the Lorenz 63 model at two different time steps. Figure 6 shows $p^{M}\left(\boldsymbol{y}_{t} \mid \boldsymbol{y}_{1: t-1}\right)$ with $M=2^{14}$ particles in log-scale at time $t=9601$. The approximated pdf $p^{M}\left(\boldsymbol{y}_{t} \mid \boldsymbol{y}_{1: t-1}\right)$ is compared with a Gaussian pdf and a Student's t-distribution (with $\nu=3$ ), all of them with the same mean and variance. Figure 7 shows the same distributions at a different time step $(t=10201)$. Note that $p^{M}\left(\boldsymbol{y}_{t} \mid \boldsymbol{y}_{1: t-1}\right)$ has very light tails at both time steps, and therefore, the assumption $(\mathfrak{C})$ holds in both numerical examples.

\section{B. Non-linear Growth Model with Heavy-tailed \\ Observation Noise}

In this numerical example, we consider the problem of tracking a modifie version of the non-linear growth model in [7]. The state and observation equations are given by

$$
X_{t}=\frac{X_{t-1}}{2}+\frac{25 X_{t-1}}{1+X_{t-1}^{2}}+8 \cos (\phi t)+U_{t},
$$
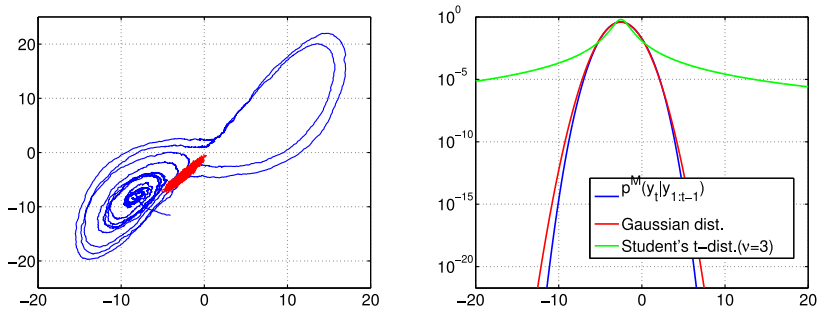

Fig. 7. Approximated observation predictive pdf $p^{M}\left(y_{t} \mid y_{1: t-1}\right)$, Gaussian distribution, and Student's t-distribution $(\nu=3)$ in log-scale for the stochastic Lorenz 63 example with $M=2^{14}$ particles. All distributions have the same mean and variance.
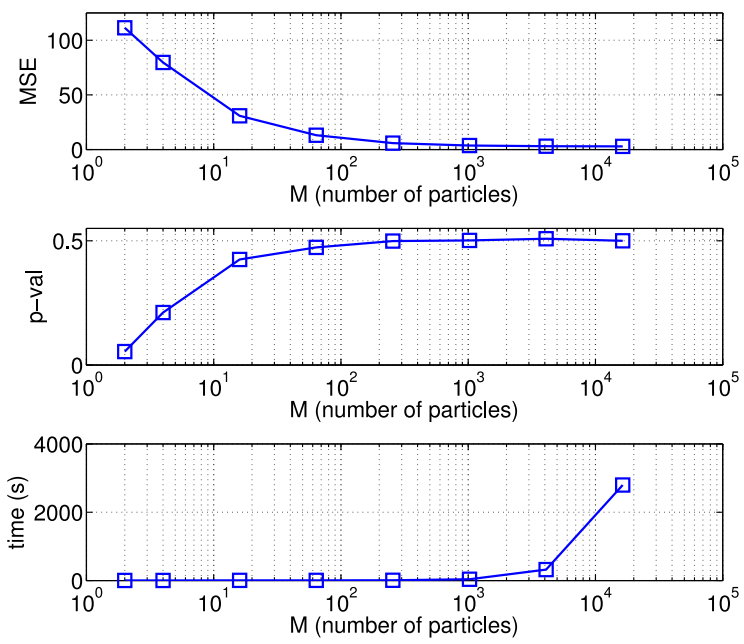

Fig. 8. BPF applied to a stochastic growth model with Student's t-distributed noise, and with fi ed number of particles (Section V-B). MSE in the approximation of the posterior mean (top), averaged $p$-value (middle) and running time (bottom). The results are averaged over 50 independent simulations.

$$
Y_{t}=\frac{X_{t}^{2}}{20}+V_{t},
$$

where $\phi=0.4$ is a frequency parameter (in $\mathrm{rad} / \mathrm{s}),\left\{U_{t}\right\}_{t \geq 1}$ denotes a sequence of independent zero-mean univariate Gaussian r.v.'s with variance $\sigma_{u}^{2}=2$, and $\left\{V_{t}\right\}_{t \geq 1}$ is a sequence of independent Student's t-distributed r.v.'s with $\nu=5$ degrees of freedom. The model is run for $t=1,2, \ldots, T$, with $T=5,000$.

First, we have run the standard BPF (with a fi ed number of particles) for $M$ in the range between 2 and $2^{14}$. Figure 8 shows, for each value of the fi ed number of particles $M$, the MSE of the approximation of the posterior mean, the averaged $\mathrm{p}$-value $p^{*}$ computed in the algorithm of Table II, and the running time. As expected, the MSE decreases with the number of particles, at the expense of increasing the computational complexity of the filte . 
TABLE V

Output of the Algorithm in TABle II For a Stochastic Growth Model With Student's t-Distributed ObSERVATION Noise, With Adaptive $M$ (Section V-B). The Algorithm Parameters are Chosen as $W=15, K=1, M_{\mathrm{MAX}}=2^{14}, M_{\mathrm{Min}}=2^{6}$. We Display the MSE IN THE Approximation OF the Posterior Mean, the Average Number of Particles $M$, the Average P-Value, AND the RunNing Time

\begin{tabular}{|l|c|c|c|c|c|c|}
\hline$\left[p_{l}-p_{h}\right]$ & {$[0.4-0.8]$} & {$[0.35-0.75]$} & {$[0.3-0.7]$} & {$[0.3-0.65]$} & {$[0.25-0.65]$} & {$[0.2-0.6]$} \\
\hline \hline MSE & 2.8707 & 3.4945 & 4.7687 & 9.0465 & 10.5826 & 17.6967 \\
\hline $\bar{M}$ & 9739 & 7478 & 6251 & 3168 & 2087 & 232 \\
\hline p-val & 0.4976 & 0.4950 & 0.4893 & 0.4837 & 0.4730 & 0.4604 \\
\hline exec. time (s) & 3613 & 2515 & 1427 & 561 & 234 & 21 \\
\hline
\end{tabular}

Note also that, over a certain range of $M$ (namely, $M \geq 2^{5}$ ), the performance does not significantl improve. Finally, we see that in this example when the performance is poor, the p-value is very low (in average). This p-value is increased to $\approx 0.5$ when the performance of the filte improves.

Then, we ran the particle filte with adaptive number of particles in Table II, with $K=5$ fictitiou observations, window size $W=15, \mathrm{p}$-value thresholds $\left[p_{l}-p_{h}\right] \in$ $\{[0.4-0.68],[0.35-0.75],[0.3-0.7],[0.3-0.65],[0.25-$ $0.65],[0.2-0.6]\}$, initial number of particles $M_{0}=2^{9}$, maximum and minimum number of particles $M_{\max }=2^{14}$ and $M_{\min }=2^{4}$, respectively, $f_{\text {up }}\left(M_{n-1}\right)=2 M_{n-1}$, and $f_{\text {down }}\left(M_{n-1}\right)=M_{n-1} / 2$.

Table V displays the MSE of the approximation of the posterior mean, the averaged number of particles, the average p-value, and the running time in seconds, for the different choices of $\left[p_{l}-p_{h}\right]$. The results are averaged over 50 independent trials. Again, the pair of thresholds $\left[p_{l}-p_{h}\right]$ allows to operate at different complexity-performance regimes; decreasing the pair of parameters worsens the performance of the filte but enables a reduction in computational load.

\section{CONCLUSION}

In practice, the number of particles needed in a particle filte is usually determined in an ad hoc manner. Furthermore, this number is typically kept constant throughout tracking. In this paper, we have proposed a methodology for the online determination of the number of particles needed by the filte. The approach is based on assessing the convergence of the predictive distribution of the observations online. First we have proved, under standard assumptions, a novel convergence result on the approximation of this distribution. Then, we have proposed a method for adapting the number of particles based on the online assessment of the filte convergence. We have illustrated the performance of the suggested algorithm by computer simulations. The proposed procedure is simple but not unique. Namely, with the proposed methodology one can develop a range of algorithms for adapting the number of particles. Furthermore, while the analysis and examples have been presented for the standard bootstrap particle filte for simplicity and clarity, it is straightforward to extend it to more sophisticated algorithms using adaptive proposals [41] or parallelization schemes [42], [43].

\section{APPENDIX A}

\section{PROOF OF PROPOSITION 2}

The sequence of r.v.'s $\left\{A_{K, t}\right\}_{t \geq 1}$ are constructed to be independent. To see this, let us look into the generation of $A_{K, t}$ and
$A_{K, t+1}$. Below, we are using capital letters to denote a r.v. (e.g., $Y_{t}$ ) and lower-case letters for its realisations (e.g., $y_{t}$ ).

At time $t$, the r.v. $A_{K, t}$ is constructed by means of a nonlinear transformation of the r.v.'s $Y_{t}$ and $\left\{\tilde{Y}_{t}^{(k)}\right\}_{k=1, \ldots, K}$. The latter are referred to as fictitiou observations in the paper. Let us denote this many-to-one transformation as $\psi$, i.e.,

$$
A_{K, t}=\psi\left(Y_{t}, \tilde{Y}_{t}^{(1)}, \ldots, \tilde{Y}_{t}^{(K)}\right) .
$$

Under the sole assumption that $\left\{Y_{t}, \tilde{Y}_{t}^{(1)}, \ldots, \tilde{Y}_{t}^{(K)}\right\}$ are i.i.d. continuous r.v.'s, Proposition 1 states that $A_{K, t}$ has a uniform probability distribution. To be precise, $A_{K, t}$ takes values on $\{0, \ldots, K\}$, and its probability mass function is $P\left(A_{K, t}=\right.$ $n)=\frac{1}{K+1}$ for every $n \in\{0, \ldots, K\}$.

In our case, the common pdf of the r.v.'s $\left\{Y_{t}, \tilde{Y}_{t}^{(1)}\right.$, $\left.\ldots, \tilde{Y}_{t}^{(K)}\right\}$ is $p_{t}\left(y_{t}\right)=\int g_{t}\left(y_{t}, \boldsymbol{x}\right) \xi_{t}(d \boldsymbol{x})=p\left(y_{t} \mid y_{1: t-1}\right)$. However, the actual form of $p\left(y_{t} \mid y_{1: t-1}\right)$ plays no role whatsoever in Proposition 1. In other words, $A_{K, t}$ is uniform as long as $\left\{Y_{t}, \tilde{Y}_{t}^{(1)}, \ldots, \tilde{Y}_{t}^{(K)}\right\}$ are i.i.d. and this results holds independently of the actual sequence $y_{1: t-1}$ (which determines the form of $p\left(y_{t} \mid y_{1: t-1}\right)$ ).

We move on to time $t+1$. The r.v. $A_{K, t+1}$ is obtained as a nonlinear transformation of $\left\{Y_{t+1}, \tilde{Y}_{t+1}^{(1)}, \ldots, \tilde{Y}_{t+1}^{(K)}\right\}$, namely,

$$
A_{K, t+1}=\psi\left(Y_{t+1}, \tilde{Y}_{t+1}^{(1)}, \ldots, \tilde{Y}_{t+1}^{(K)}\right) \text {. }
$$

From Proposition 1, if $\left\{Y_{t+1}, \tilde{Y}_{t+1}^{(1)}, \ldots, \tilde{Y}_{t+1}^{(K)}\right\}$ are i.i.d. then $A_{K, t+1}$ has a uniform distribution, i.e., $P\left(A_{K, t+1}=n\right)=\frac{1}{K+1}$ for every $n \in\{0, \ldots, K\}$. As before, this is true independently of the specifi common pdf of the r.v.'s $\left\{Y_{t+1}, \tilde{Y}_{t+1}^{(1)}, \ldots, \tilde{Y}_{t+1}^{(K)}\right\}$. This common pdf is $p_{t+1}\left(y_{t+1}\right)=\left(g_{t+1}^{y_{t+1}}, \xi_{t+1}\right)=p\left(y_{t+1} \mid y_{1: t}\right)$ and, therefore, $A_{K, t+1}$ is uniform without regard to the sequence $y_{1: t}$ (which determines the form of $p\left(y_{t+1} \mid y_{1: t}\right)$ ) and, in particular, without regard to the observed realisation $Y_{t}=y_{t}$.

Now, since $A_{K, t+1}$ is uniform for any $Y_{t}=y_{t}$ (and, obviously, for any $\left.\tilde{Y}_{t}^{(k)}=\tilde{y}_{t}^{(k)}, k=1, \ldots, K\right)$, and $A_{K, t}$ is obtained as a transformation of $\left\{Y_{t}, \tilde{Y}_{t}^{(1)}, \ldots, \tilde{Y}_{t}^{(K)}\right\}$ (see (21) above), then it follows that $A_{K, t+1}$ has a uniform distribution for every possible realisation $A_{K, t}=n$. This implies that the conditional distribution of $A_{K, t+1}$ given $A_{K, t}$ is uniform, i.e.,

$$
P\left(A_{K, t+1}=n \mid A_{K, t}=m\right)=\frac{1}{K+1},
$$

$\forall(n, m) \in\{0, \ldots, K\} \times\{0, \ldots, K\}$. However, Eq. (22) readily entails independence. If we let $P\left(A_{K, t+1}, A_{K, t}\right)$ denote the joint probability mass function of $A_{K, t+1}$ and $A_{K, t}$, then from 
the definitio of conditional probability we have

$$
\begin{array}{r}
P\left(A_{K, t+1}=n, A_{K, t}=m\right)= \\
P\left(A_{K, t+1}=n \mid A_{K, t}=m\right) P\left(A_{K, t}=m\right)= \\
\frac{1}{K+1} \times \frac{1}{K+1}= \\
P\left(A_{K, t+1}=n\right) P\left(A_{K, t}=m\right),
\end{array}
$$

for any $n$ and $m$ within the set $\{0, \ldots, K\}$.

\section{APPENDIX B}

\section{PROOF OF THEOREM 2}

Let $Y_{t}$ denote the (random) observation at time $t$. Assume, without loss of generality, that $\mathcal{Y}=\mathbb{R}$. The probability measure associated to $Y_{t} \mid Y_{1: t-1}=y_{1: t-1}$ is $\mu_{t}(d y)$ and, therefore, we can write the cumulative distribution function of $Y_{t} \mid Y_{1: t-1}=y_{1: t-1}$ as $F_{t}(z)=\left(I_{(-\infty, z]}, \mu_{t}\right)$, where

$$
I_{A}(y)= \begin{cases}1, & \text { if } y \in A \\ 0, & \text { otherwise }\end{cases}
$$

is the indicator function. Obviously, $\left\|I_{A}\right\|_{\infty}=1<\infty$ independently of the set $A$ and, therefore, Theorem 1 yields

$$
\lim _{M \rightarrow \infty} F_{t}^{M}(z)=F_{t}(z) \text { a.s. }
$$

for any $z \in \mathbb{R}$, where $F_{t}^{M}(z)=\left(I_{(-\infty, z]}, \mu_{t}^{M}\right)$ is the approximation of the cdf of $Y_{t} \mid Y_{1: t-1}=y_{1: t-1}$ provided by the BPF.

Assume the actual observation is $Y_{t}=y_{t}$ and we draw $K$ i.i.d. fictitiou observations $\tilde{y}_{t}^{(1)}, \ldots, \tilde{y}_{t}^{(K)}$ from the distribution with cdf $F_{t}^{M}$. Given $Y_{t}=y_{t}$ is fi ed, the probability that exactly $n$ out of $K$ of these samples are lesser than $y_{t}$ coincides with the probability to have $n$ successes out of $K$ trials for a binomial r.v. with parameter (i.e., success probability) $F_{t}^{M}\left(y_{t}\right)$, which can be written as

$$
h_{n}^{M}\left(y_{t}\right)=\left(\begin{array}{cc}
K & \left(F_{t}^{M}\left(y_{t}\right)\right)^{n}\left(1-F_{t}^{M}\left(y_{t}\right)\right)^{K-n} .
\end{array}\right.
$$

By integrating $h_{n}^{M}\left(y_{t}\right)$ over the predictive distribution of $Y_{t}$, we obtain the probability to have exactly $n$ fictitiou observations, out of $K$, which are less than the r.v. $Y_{t}$, i.e., the probability that $A_{K, M, t}=n$ is

$$
\mathbb{Q}_{K, M, t}(n)=\left(h_{n}^{M}, \mu_{t}\right) .
$$

However, Theorem 1 yields $\lim _{M \rightarrow \infty}\left(h_{n}^{M}, \mu_{t}^{M}\right)=\left(h_{n}^{M}, \mu_{t}\right)$ a.s. ${ }^{4}$ and, in particular, there exists a sequence of non-negative r.v.'s $\left\{\varepsilon_{M}\right\}_{M \geq 1}$ such that $\lim _{M \rightarrow \infty} \varepsilon_{M}=0$ a.s. and

$$
\left(h_{n}^{M}, \mu_{t}^{M}\right)-\varepsilon_{M} \leq\left(h_{n}^{M}, \mu_{t}\right) \leq\left(h_{n}^{M}, \mu_{t}^{M}\right)+\varepsilon_{M}
$$

\footnotetext{
${ }^{4}$ Note that $\left\|h_{n}^{M}\right\|_{\infty}=1$ independently of $n$ and $M$. If we recall the proof of Theorem 1 , namely inequality (38) in the supplementary material, we observe that the error rates for the approximation errors of the form $\left|\left(h, \mu_{t}^{M}\right)-\left(h, \mu_{t}\right)\right|$ depend on the test function $h$ only through its supremum $\|h\|_{\infty}$, i.e., the r.v. $\tilde{W}_{t}^{\varepsilon}$ in (38) in the supplementary material, only depends on the observations $\boldsymbol{y}_{1: t-1}$ and the model (specificall the likelihood functions). Therefore, Theorem 1 (the same as, e.g., Lemmas 1 and 2 in the supplementary material) also holds for any test function that depends on $M$ (even a random one) as long as its supremum is deterministic and independent of $M$. This is the case of function $h_{n}^{M}(y)$.
}

for each $M$. Moreover, it is apparent that $\left(h_{n}^{M}, \mu_{t}^{M}\right)=\frac{1}{K+1}$ (see Proposition 1) which, together with (24) and (25) yields the desired relationship

$$
\frac{1}{K+1}-\varepsilon_{M} \leq \mathbb{Q}_{K, M, t}(n) \leq \frac{1}{K+1}+\varepsilon_{M}
$$

for every $n \in\{0, \ldots, K\}$.

\section{REFERENCES}

[1] M. West and J. Harrison, Bayesian Forecasting, 2nd ed. New York, NY USA: Springer-Verlag, 1996.

[2] B. Ristic, S. Arulampalam, and N. Gordon, Beyond the Kalman Filter. Boston, MA, USA: Artech House, 2004.

[3] R. E. Kalman, "A new approach to linear filterin and prediction problems," J. Basic Eng., vol. 82, pp. 35-45, 1960.

[4] B. D. O. Anderson and J. B. Moore, Optimal Filtering. Mineola, NY, USA: Dover, 1979.

[5] L. R. Rabiner and B. H. Juang, "An introduction to hidden Markov models," ASSP Mag., vol. 3, no. 1, pp. 4-16, 1986.

[6] J. S. Liu, R. Chen, and W. H. Wong, "Rejection control and sequential importance sampling," J. Amer. Statist. Assoc., vol. 93, no. 443, pp. 1022 1031, Sep. 1998.

[7] A. Doucet, S. Godsill, and C. Andrieu, "On sequential Monte Carlo Sampling methods for Bayesian filtering" Statist. Comput., vol. 10, no. 3, pp. 197-208, 2000.

[8] A. Doucet, N. de Freitas, and N. Gordon, Eds., Sequential Monte Carlo Methods in Practice. New York, NY, USA: Springer, 2001.

[9] P. M. Djurić et al., "Particle filtering” IEEE Signal Process. Mag., vol. 20, no. 5, pp. 19-38, Sep. 2003.

[10] O. Cappé, S. J. Godsill, and E. Moulines, “An overview of existing methods and recent advances in sequential Monte Carlo," Proc. IEEE, vol. 95, no. 5, pp. 899-924, May 2007.

[11] N. Gordon, D. Salmond, and A. F. M. Smith, "Novel approach to nonlinear and non-Gaussian Bayesian state estimation," Proc. Inst. Electr. Eng.-F, Radar Signal Process., vol. 140, pp. 107-113, 1993.

[12] A. Bain and D. Crisan, Fundamentals of Stochastic Filtering. New York, NY, USA: Springer, 2008.

[13] P. Del Moral and L. Miclo, "Branching and interacting particle systems Approximations of Feynman-Kac formulae with applications to nonlinear filtering" in Séminaire de Probabilités XXXIV (Lecture Notes in Mathematics). Berlin, Germany: Springer-Verlag, 2000, pp. 1-145.

[14] D. Fox, "Adapting the sample size in particle filter through KLDsampling," Int. J. Robot. Res., vol. 22, no. 12, pp. 985-1003, 2003.

[15] A. Soto, "Self adaptive particle filter" in Proc. Int. Joint Conf. Artif. Intell., 2005, pp. 1398-1406.

[16] O. Straka and M. Šimandl, "Particle filte adaptation based on efficien sample size,” in Proc. 14th IFAC Symp. Syst. Identificatio , 2006, pp. 991996.

[17] A. Lee and N. Whiteley, "Variance estimation and allocation in the particle filter" arXiv:1509.00394v2 [stat.CO], 2016.

[18] P. D. Moral, Feynman-Kac Formulae: Genealogical and Interacting Particle Systems With Applications. New York, NY, USA: Springer, 2004.

[19] A. Bhadra and E. L. Ionides, "Adaptive particle allocation in iterated sequential Monte Carlo via approximating meta-models," Statist. Comput., vol. 26 , no. $1 / 2$, pp. 393-407, 2016

[20] F. LeGland and N. Oudjane, "A sequential particle algorithm that keeps the particle system alive," in Proc. 13th Eur. Signal Process. Conf., 2005, pp. $1-4$.

[21] A. Jasra, A. Lee, C. Yau, and X. Zhang, "The alive particle filte ," arXiv:1304.0151, 2013

[22] P. D. Moral, A. Jasra, A. Lee, C. Yau, and X. Zhang, "The alive particle filte and its use in particle Markov chain Monte Carlo," Stochastic Anal. Appl., vol. 33, no. 6, pp. 943-974, 2015.

[23] X. Hu, T. Schon, and L. Ljung, "A basic convergence result for particle filtering” IEEE Trans. Signal Process., vol. 56, no. 4, pp. 1337-1348, Apr. 2008.

[24] J. Cornebise, "Adaptive sequential Monte Carlo methods," Ph.D. dissertation, Télécom ParisTech, Paris, France, 2010.

[25] A. Beskos, D. Crisan, and A. Jasra, "On the stability of sequential monte carlo methods in high dimensions," Ann. Appl. Probability, vol. 24, no. 4 pp. 1396-1445, 2014 
[26] P. M. Djurić and J. Míguez, "Assessment of nonlinear dynamic models by Kolmogorov-Smirnov statistics," IEEE Trans. Signal Process., vol. 58, no. 10, pp. 5069-5079, Oct. 2010.

[27] R. Chen, X. Wang, and J. S. Liu, "Adaptive joint detection and decoding in flat- ading channels via mixture Kalman filtering" IEEE Trans. Inf. Theory, vol. 46, no. 6, pp. 2079-2094, Sep. 2000.

[28] N. Chopin, P. E. Jacob, and O. Papaspiliopoulos, "SMC2: An efficien algorithm for sequential analysis of state space models," J. Roy. Statist. Soc., Series B (Statist. Methodol.), vol. 75, no. 3, pp. 397-426, 2012.

[29] D. Crisan and J. Miguez, "Nested particle filter for online parameter estimation in discrete-time state-space Markov models," arXiv:1308.1883v4 [stat.CO], 2016.

[30] C. Andrieu, A. Doucet, and R. Holenstein, "Particle Markov chain Monte Carlo methods," J. Roy. Statist. Soc. B, vol. 72, no. 3, pp. 269-342, 2010

[31] E. Koblents and J. Míguez, "A population Monte Carlo scheme with transformed weights and its application to stochastic kinetic models," Statist. Comput., vol. 25, no. 2, pp. 407-425, 2015.

[32] A. Doucet, N. de Freitas, and N. Gordon, "An introduction to sequential Monte Carlo methods," in Sequential Monte Carlo Methods in Practice, A. Doucet, N. de Freitas, and N. Gordon, Eds. New York, NY, USA: Springer, 2001, ch. 1, pp. 4-14.

[33] J. Carpenter, P. Clifford, and P. Fearnhead, "Improved particle filte for nonlinear problems," Proc. Inst. Electr. Eng.-Radar, Sonar Navigat., vol. 146, no. 1, pp. 2-7, Feb. 1999.

[34] D. Crisan, "Particle filters - theoretical perspective," in Sequential Monte Carlo Methods in Practice, A. Doucet, N. de Freitas, and N. Gordon, Eds. New York, NY, USA: Springer, 2001, ch. 2, pp. 17-42.

[35] T. Li, M. Bolić, and P. M. Djurić, "Resampling methods for particle filtering” IEEE Signal Process. Mag., vol. 32, no. 3, pp. 70-86, May 2015.

[36] K. Heine and D. Crisan, "Uniform approximations of discrete-time filters" Adv. Appl. Probability, vol. 40, no. 4, pp. 979-1001, 2008.

[37] R. L. Plackett, "Karl Pearson and the chi-squared test," Int. Statist. Rev., pp. 59-72, 1983.

[38] E. N. Lorenz, "Deterministic nonperiodic fl w," J. Atmospheric Sci., vol. 20, no. 2, pp. 130-141, 1963.

[39] A. J. Chorin and P. Krause, "Dimensional reduction for a Bayesian filter" Proc. Nat. Acad. Soc., vol. 101, no. 42, pp. 15013-15017, Oct. 2004.

[40] M. S. Nikulin, "Hellinger distance," Encyclopedia of Mathematics. New York, NY, USA: Springer, 2001.

[41] J. Cornebise, E. Moulines, and J. Olsson, "Adaptive methods for sequential importance sampling with application to state space models," Statist. Comput., vol. 18, no. 4, pp. 461-480, 2008.

[42] N. Whiteley, A. Lee, and K. Heine, "On the role of interaction in sequential monte carlo algorithms," Bernoulli, vol. 22, no. 1, pp. 494-529, 2016.

[43] B. Paige, F. Wood, A. Doucet, and Y. W. Teh, "Asynchronous anytime sequential Monte Carlo," in Proc. Adv. Neural Inform. Process. Syst., 2014, pp. 3410-3418. 